\title{
Why Major Programs Need Innovation Support Labs: An Example from the Space Shuttle Launch Program at KSC
}

\author{
Robert C. Youngquist ${ }^{1}$, Stanley O. Starr ${ }^{2}$, Charles G. Stevenson ${ }^{3}$, Jorge E. Rivera ${ }^{4}$, and Steven J. Sullivan ${ }^{5}$ \\ NASA, Kennedy Space Center, Florida, 32899
}

\begin{abstract}
For over 30 years the Kennedy Space Center (KSC) has processed the Space Shuttle; handling all hands-on aspects from receiving the Orbiter, External Tanks, Solid Rocket Booster Segments, and Payloads, through certification, check-out, and assembly, and ending with fueling, count-down, and launch. A team of thousands have worked this highly complicated, yet supremely organized, process and have, as a consequence, generated an exceptional amount of technology to solve a host of problems. This paper describes the contributions of one team that formed with the express purpose to help solve some of these diverse Shuttle ground processing problems.
\end{abstract}

\section{Introduction}

$\mathrm{G}$ round processing of the Space Shuttle has occurred at the Kennedy Space Center for over 30 years. This activity has encompassed everything from safing an Orbiter just after landing to launching the completed stack from one of two launch pads. It includes demating an Orbiter from the 747 when it flies in from the west coast, moving External Tanks (ET) from barges to the Vehicle Assembly Building (VAB), and receiving Solid Rocket Booster (SRB) segments from rail cars. It includes detailed processing of an Orbiter in one of three Orbiter Processing Facilities (OPF) to return it to a safe launching configuration, and then within the VAB, stacking the SRB segments, hanging an ET from these SRBs, and finally forming a complete stack by adding the Orbiter; forming a complete Space Shuttle Vehicle. It includes maintenance and processing of launch pads, handling of cryogenic fuels and hypergols, and monitoring of thousands of sensors and cameras needed to ensure that all is well. Finally, it includes safely launching the vehicle.

Performing these processing activities requires thousands of trained technicians, engineers, and managers working in a highly organized environment, ensuring that every critical step is performed and properly documented; and with such an intelligent and motivated team comes innovation. There are hundreds of documented cases of KSC employees not only suggesting improvements to processes, but implementing them, sometimes building the hardware in their home garages. In some instances these improvements arose because new supportive technologies became available (a clear example here is the impact of ever increasing computer power). In others, advances occurred because new requirements were levied on the program and innovation was required to meet these. And finally, sometimes improvements to processes resulted from just shear imagination and insight; truly creative acts.

Trying to document the totality of innovation generated by the Space Shuttle team at the Kennedy Space Center is beyond the scope of this paper, so we will limit the discussion to a small, but highly productive, subset, namely the interaction between the KSC Applied Physics Laboratory (APL) and the KSC Shuttle Ground Processing Directorate. The KSC APL is composed of about 8 NASA civil servants (as well as a varying number of contractors) with roughly 200 total years of KSC experience. The proximity of this lab to the KSC Shuttle workforce allowed regular interactions with engineers and technicians, as well as safety and reliability personnel, alleviating many of the hardware implementation problems experienced by development labs located at other centers. As a result, numerous pieces of Ground Support Equipment and Shop Aids were generated by the KSC APL that solved

\footnotetext{
${ }^{1}$ Physicist/Lab Lead, Applied Physics Branch, Materials Science Division, Mail Stop NE-L5

${ }^{2}$ Physicist/Branch Chief, Applied Physics Branch, Materials Science Division, Mail Stop NE-L5

${ }^{3}$ Shuttle Ground Processing Engineer, Engineering Support, Mail Stop ESC-600

${ }^{4}$ Deputy Flight and Ground Processing Chief Engineer, Operational Engineering Systems Office, Mail Stop NE-O

${ }^{5}$ Flight and Ground Processing Chief Engineer, Operational Engineering Systems Office, Mail Stop NE-O
} 
problems, increased safety, and improved the overall processing of the vehicles and their components. In addition, the lab generated numerous single-use prototypes to resolve immediate issues or to demonstrate capability.

The KSC APL also acted as a conduit between KSC Shuttle operations and the more than $200 \mathrm{KSC}$ engineers and scientists who were not directly tied to the Shuttle Program. When problems arose that could be routed to KSC areas of expertise outside of the KSC APL, such as corrosion, cryogenics, advanced modeling, mechanics, sensor qualification, and others, the lab would make the appropriate contacts to arrange for an effective response. But sometimes problems would arise that were outside of the realm of KSC expertise and in those cases the lab would contact other centers, industry, and universities. Using NASA's small business technology transfer (STTR) program and its graduate students researchers program (GSRP) the lab has coordinated the demonstration of advanced hydrogen detection technology, active shock isolation for the mobile launch platform, and, perhaps most significant, a wireless, passive, sensor network.

The interaction between Shuttle Ground Processing and the KSC APL serves as a model for the two way interchange between an active launch support program and an extended engineering/science capability. On the Shuttle operations side an effective mechanism was developed where problems experienced by technicians and engineers could be brought to a few individuals, who would then bring those problems to KSC APL personnel. On the lab side care was taken not to become specialists, but to represent a diverse range of areas so that an appropriate, not a favorite, technology be presented to help resolve a problem. As a result of this relationship, significant hardware was delivered, many NASA Tech Briefs were published, several patents awarded (with commercialization), and novel technologies developed that will impact future vehicle ground support programs.

\section{Overview}

The KSC Applied Physics Laboratory (APL) has worked closely with Space Shuttle Ground Support for over 20 years, resulting in the delivery of numerous pieces of hardware, significant insights and analyses, and, in several cases, concepts that impacted lives outside of the Shuttle Program. This paper highlights the most significant accomplishments of the lab and is broken into five sections based loosely on technology area.

In the late 1980's through the 1990's the lab's "bread and butter" product-line was alignment and positioning aids for the launch elements - mainly the External Tank. The majority of these tools were custom built systems designed to address specific positioning or alignment problems, but one small, hand-held instrument, the scaling device, became commercialized and was eventually used as a crime scene aid and helped to solve murder cases in Iraq.

Then, after establishing a reputation with the External Tank group, the lab branched out to address other Shuttle related needs and soon gained a reputation for being able to develop a wide range of innovative hardware, but with specific expertise in optical systems. The second section describes our contributions in this area ranging from relatively simple optical inspection tools to state-of-the-art Orbiter window defect location and inspection systems. Within this technical area tracking software was developed to aid the Columbia Accident Investigation Board (CAIB) and this software passed through a series of applications, ending with its use to dimension earthquake damaged structures in Haiti.

The third section discusses the impact that water, in various forms, has had on the KSC APL deliveries to the Space Shuttle Program. One problem area started with water being trapped in Shuttle tiles, causing us to develop and deliver sensors to find wet tiles and blankets as well as a Water Extraction Tool (WET) to remove water from the tiles. In addition we significantly supported the testing and development of an ice imaging camera and the establishment of hail sensors used to support Space Shuttle launches.

The fourth section reflects the lab's ability to develop sensors and these cover a diverse area of requests. Starting with innovations in the 1990's in ultrasonic leak detection and ultraviolet fire detector support hardware - both of which have been patented-the KSC APL was called upon numerous times to develop innovative sensors. A method for measuring over-center latch position for the Orbiter robotic arm was created as well as a novel nonintrusive device to monitor GN2 flow within stainless steel tubing. We also generated several techniques for assessing the health of the large liquid hydrogen tanks located at the launch pads; providing insight into their interior support system, the status of their internal insulation, and an indication of air leaks. 
And finally, the fifth section presents two major developments that were outside of the expertise of the KSC work force and were sent out as Small Business Technology Transfer (STTR) projects with Graduate Student Research Program (GSRP) support. One of these projects led to the successful demonstration of two different technologies to provide vibration/shock isolation to racks of equipment in the Mobile Launch Platforms (MLP). The other, perhaps the most significant of all the KSC Space Shuttle related innovations, is the development of a wireless surface acoustic wave multiplexed sensor system, a possible precursor to ubiquitous, inexpensive sensing.

\section{A. Alignment and Positioning Aids}

The KSC Applied Physics Laboratory started in the early 1990's and the first problem brought to the lab by the ground support team (specifically two of the authors, Charles Stevenson and Jorge Rivera) was to develop a tool that could be used to position, at its aft end, the External Tank (ET) between the two Solid Rocket Boosters (SRB). In practice the forward end of the ET is first attached to the SRBs and the aft end is not locked down until it has been positioned accurately both side to side as well as up/down (from the pilot's perspective). However, positioning the External Tank was challenging. Referring to Fig. 1, the gap between the ET and the SRBs is relatively small (typically 10-12 inches) and access is difficult. This measurement was being performed by placing personnel into slings and suspending them near the gap where they would use large calipers to reach between the flight articles. Not only was this somewhat hazardous to the suspended worker, but it increased the possibility of damaging either the ET foam or the SRB cork.
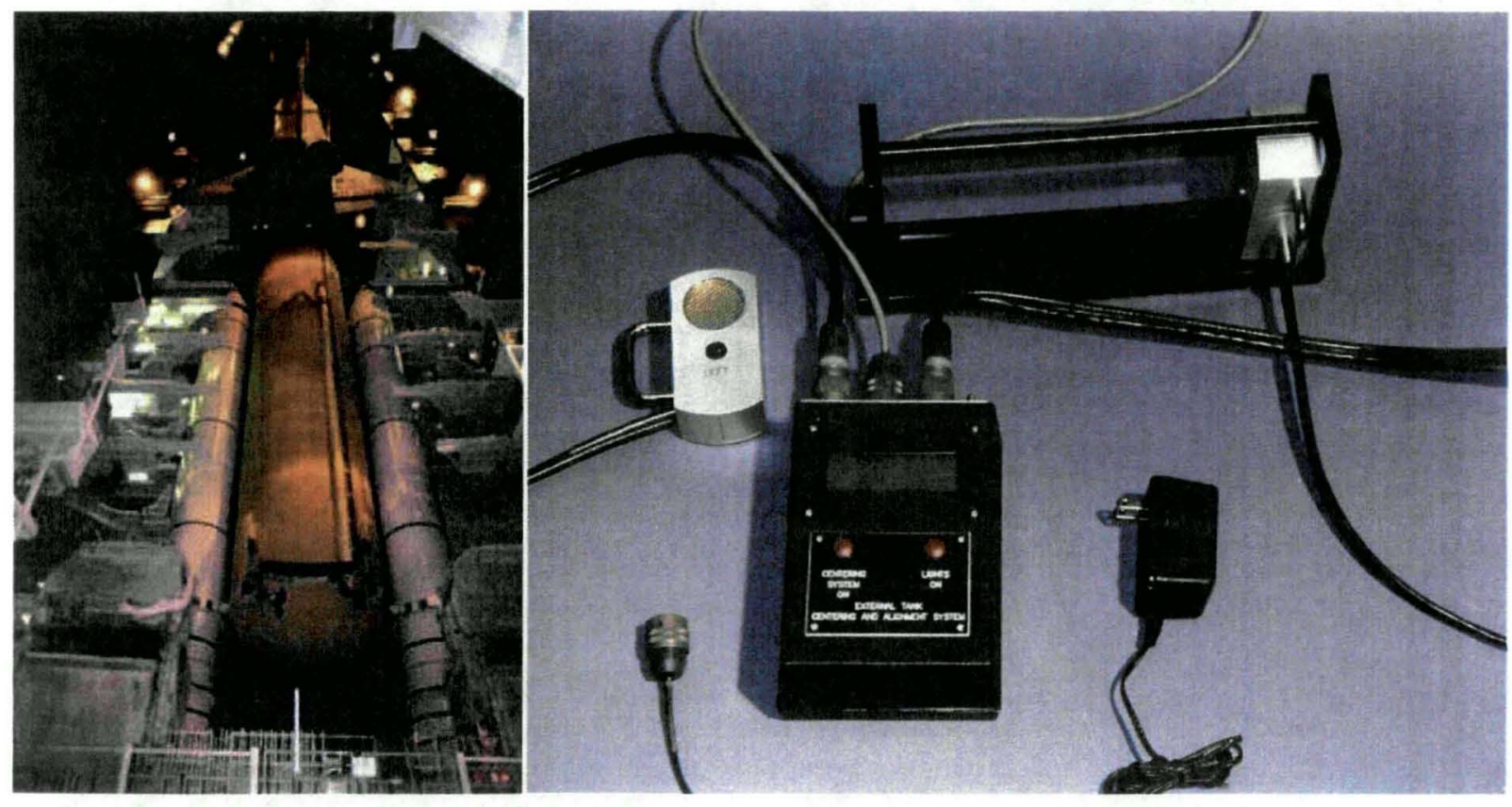

Figure 1. The External Tank Centering and Alignment System. The first major deliverable to the KSC Space Shuttle Program from the Applied Physics Lab was a system that measured the gaps between the Solid Rocket Boosters and the External Tank at the aft end.

The Applied Physics Laboratory developed a novel tool to perform this measurement', eliminating the need for direct human involvement. The tool is shown on the right hand side of Fig. 1 and consists of a pair of small metal modules that magnetically attach to the Solid Rocket Boosters. These devices contain a laser beam projector and an ultrasonic transmitter/receiver. They are attached to the SRBs when the platforms are still in place, before the External Tank has been lowered onto the forward mating fixtures. Then, after the ET has been brought in, the lasers are turned on, producing spots on the ET foam that allow it to be aligned with the SRBs. The ultrasonic transducers are used to measure the distance from the SRBs to the ET foam and a battery operated, remote module, shown in the 
figure, displays the gap distances between each SRB and the ET as well as the adjustment distance needed to center the ET. This, "External Tank Centering and Alignment System," was well received and was converted to Ground Support Equipment (GSE), allowing it to be used as the primary means for meeting this positioning requirement.
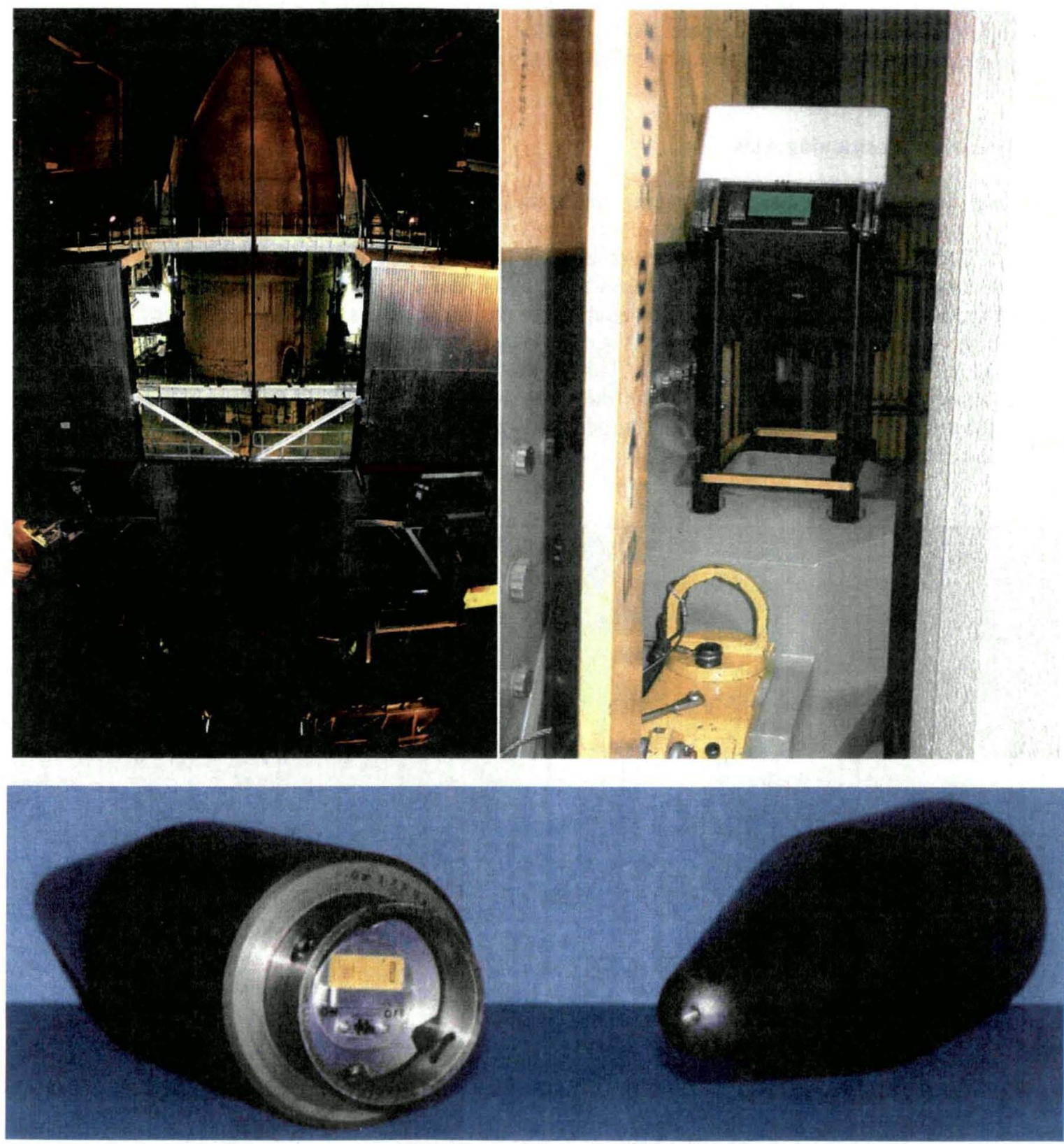

Figure 2. The External Tank Taper Pin Aft Alignment System. A laser based tool was developed to help align fixtures on the forward end of the External Tank to their mates on the Solid Rocket Boosters. Lasers were installed into taper pins, shown in the lower image, located on the SRB fixture, which launched a laser beam up onto a target mounted to the External Tank, shown in the image on the right. This system was subsequently also used for demate operations; the image on the left shows preparations being made to demate the ET from the SRBs. For demate operations it was desirable to see into the mating fixture so a wireless camera module was added to the tool as shown in the image on the right.

The ET Centering and Alignment System was one of the first tools incorporated into Shuttle Ground Processing that contained a laser. Obtaining permission to use this device, even though it was only Class II, required 
interactions with Safety personnel, discussions, and paperwork, but once these hurdles were passed it opened the door for other laser based tools. The next request made to the KSC APL was to develop a tool that could be used to position the External Tank relative to the Solid Rocket Boosters while it was suspended from a crane 30 or more feet above its final position. The problem here was one of perspective, it was difficult to visually determine when the ET was in the correct position and orientation to be lowered in between the SRBs. Consequently, the resulting iterative process of lateral and rotary adjustments, followed by small drops in height, took excessive time and was fraught with the possibility of contact between these fragile structures.

One of the authors, Jorge Rivera, noticed that the SRB mating fixture taper pins - used to help guide the ET fixtures onto the SRB fixture - were hollow and had a through hole on their top point (see the lower image in Fig. 2). Rivera suggested that if a laser were installed inside of each taper pin that the resulting beam would project vertically, providing an alignment indicator for the ET. He then suggested that a target be constructed that could be mounted over the ET mating fixture, see the image on the right hand side of Fig. 2, so that when the laser beam hit the target the offset of the ET could be quickly determined and adjustments made. This hardware was constructed and quickly implemented by the Shuttle Ground Processing Team, providing a significant enhancement in the time and safety required to mate the ET to the SRBs. In addition, several years later, the system was upgraded with the installation of wireless cameras, located in the white box shown in the upper right image of Fig. 2. These allowed move conductors to monitor the side loading of the ET during demating operations (see the left image in Fig. 2).
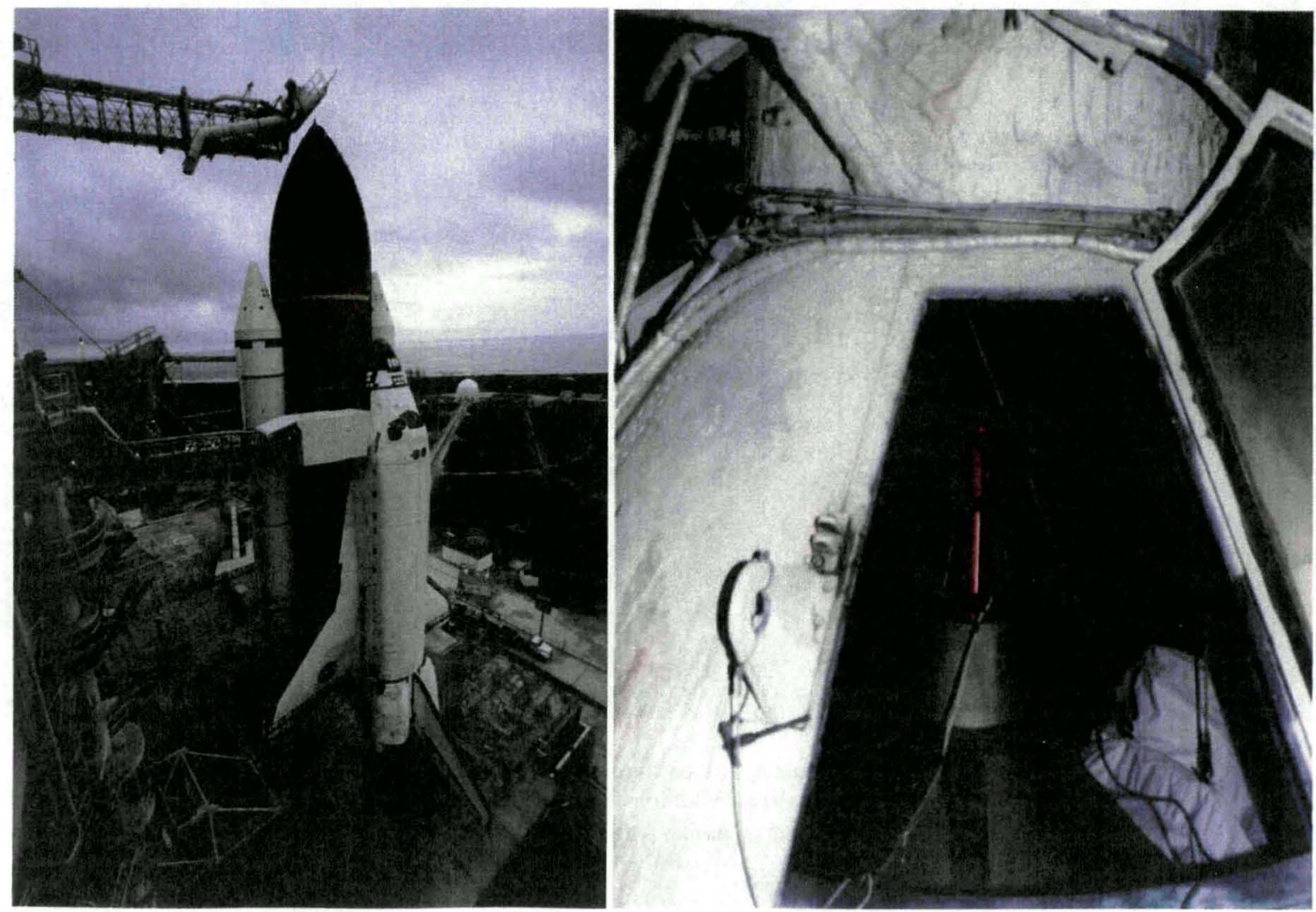

Figure 3. The GOX Vent Hood Alignment Tool. The left image shows the gaseous oxygen (GOX) vent hood lifted above the External Tank. The right image shows the GOX Vent Hood Alignment Tool (the red object mounted on the top of the External Tank), a device used to position the vent hood properly relative to the External Tank.

After the Space Shuttle is transported to the launch pad, the Gaseous Oxygen (GOX) vent hood is positioned and brought down onto the top of the External Tank. This GOX vent hood is used to capture the liquid oxygen boil off that occurs while filling the ET and direct the GOX away from the Space Shuttle. Consequently, it is important that the GOX vent hood be properly positioned relative to the ET to minimize leakage, yet, in the past, this required a 
survey team up to a full day to accomplish. The KSC Applied Physics Lab was asked to develop an alternative positioning device to alleviate the delays associated with surveying. The result was another laser-ultrasonic device, similar to the ET Centering and Alignment System. A laser beam is projected directly upward, indicating the center of the GOX vent hood, while an ultrasonic unit measures the distance. This device, shown in Fig. 3, was readily accepted and inserted into the launch processing procedure.
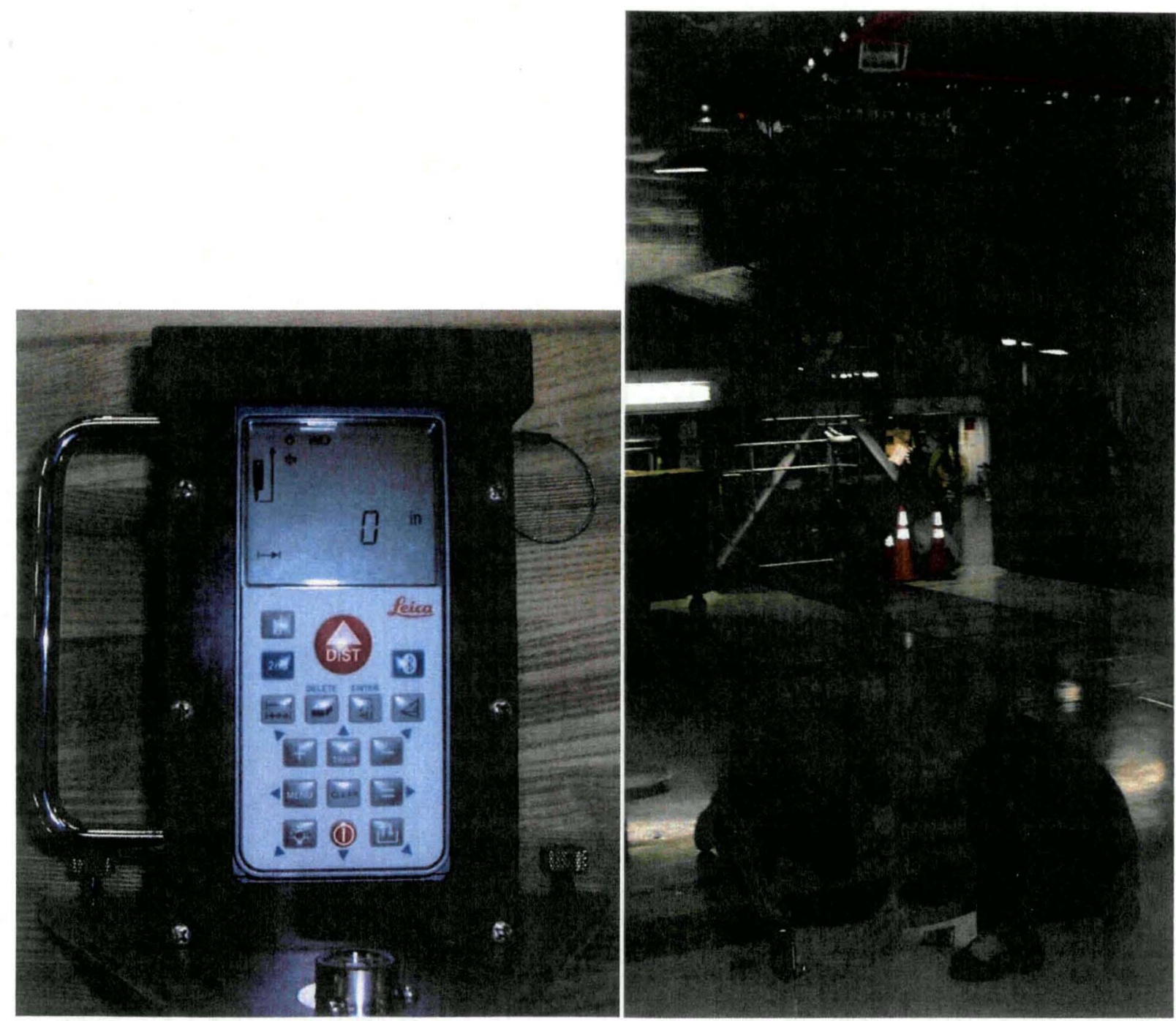

Figure 4. The Orbiter Height and Level Measurement System. The Orbiter Height and Level Measurement System was developed to ensure that the Orbiter was in the correct position after being lifted by jacks in one of the Orbiter Processing Facilities (OPF). This system went through various upgrades and was eventually made wireless, becoming the first use of Bluetooth within an OPF.

As the lab's reputation grew requests for support began to come in from the Orbiter portion of Shuttle Ground Processing. One of the earliest was to develop a system that could measure the height above the floor to four corner locations on the underside of the Orbiter within one of the Orbiter Processing Facilities (OPF). The Orbiter, after being brought into the OPF, is carefully positioned and then lifted, using jacks, to a desired height where various platforms and assorted hardware can access it. The position and orientation are critical since errors could cause contact or prevent an interface from reaching a desired location on the vehicle. In the past the height and orientation of the Orbiter were determined using manually extended telescoping measuring sticks, subject to various human errors such as compression against the vehicle, tilt, sag, and bowing. 
The KSC Applied Physics Laboratory solved this problem by adopting a laser based distance monitoring system it had constructed to locate the X-33 test vehicle as it backed onto its launch platform ${ }^{2,3}$. The system consists of four OEM repackaged laser distance measurement devices, one each placed on the floor beneath the desired locations at the corners of the vehicle. These devices have tilt adjust and levels, ensuring that the laser beams are pointing vertically. The first generation version of this system used laser measurement devices that were wired to a laptop computer where the height information was displayed, but several upgrades were made and the final system was wireless, representing the first use of Bluetooth in an Orbiter Processing Facility. Figure 4 shows one of the units and two Shuttle personnel installing one below the Orbiter in an OPF. While performing this work the KSC APL also delivered, to the same users, instrumentation to establish a coordinate system aligned with the Orbiter tires that would allow the center of gravity of the vehicle to be determined ${ }^{4}$.

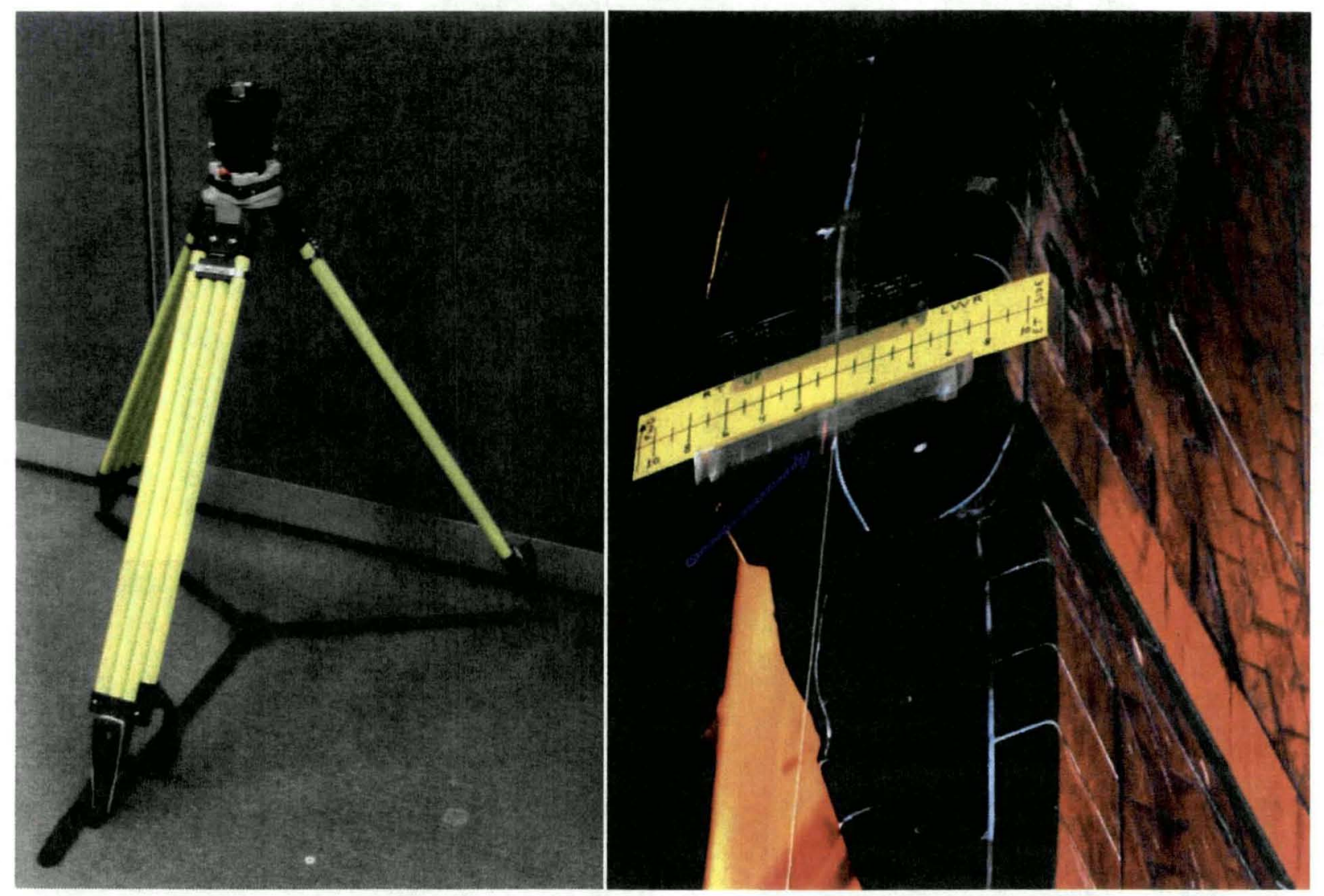

Figure 5. The Orbiter Wing Tip Alignment System. This is an image of a laser line projector designed to provide an alignment plane of light to orient the wing tips of the Orbiter. This is used to accurately set the rotation angle of the Orbiter before lowering it into the integration cell so that it can be mated to the External tank.

Another request from the Orbiter processing team was for a laser based device that could help determine the rotation angle of the Orbiter during crane operations (Figure 1 shows the Orbiter being lowered in an integration cell on its way to being attached to the External Tank.). When the Orbiter is picked up and positioned near the ceiling of the Vehicle Assembly Building over the External Tank/Solid Rocket Booster Assembly, it needs to be rotated and placed into the proper orientation before being lowered. In the past small paper rulers were attached to the wing tips of the Orbiter (see the right hand image in Fig. 5) and personnel tried to, by eye, align the ruler markings with survey spots on the nearby platforms. The goal being that the two survey spots and the centers of each wing tip be placed into the same plane, but doing this accurately by eye, with the Orbiter itself in the way, was nearly impossible. 
The KSC Applied Physics Laboratory solved this problem by mounting laser line projectors onto survey tripods (see the left hand image in Fig. 5). These devices are located directly over the survey marks on the platforms before the Orbiter is brought into the integration cell. Adjustments allow the laser lines to be aligned until they pass through each other (each is aimed at the survey mark on the opposite platform) forming a single vertical sheet of light. Then, when the Orbiter is brought in, the laser lines appear on the wing tip rulers (see the right hand image in Fig. 5), indicating how the Orbiter should be adjusted to properly orient it. This system was readily accepted by the Orbiter Processing Team.
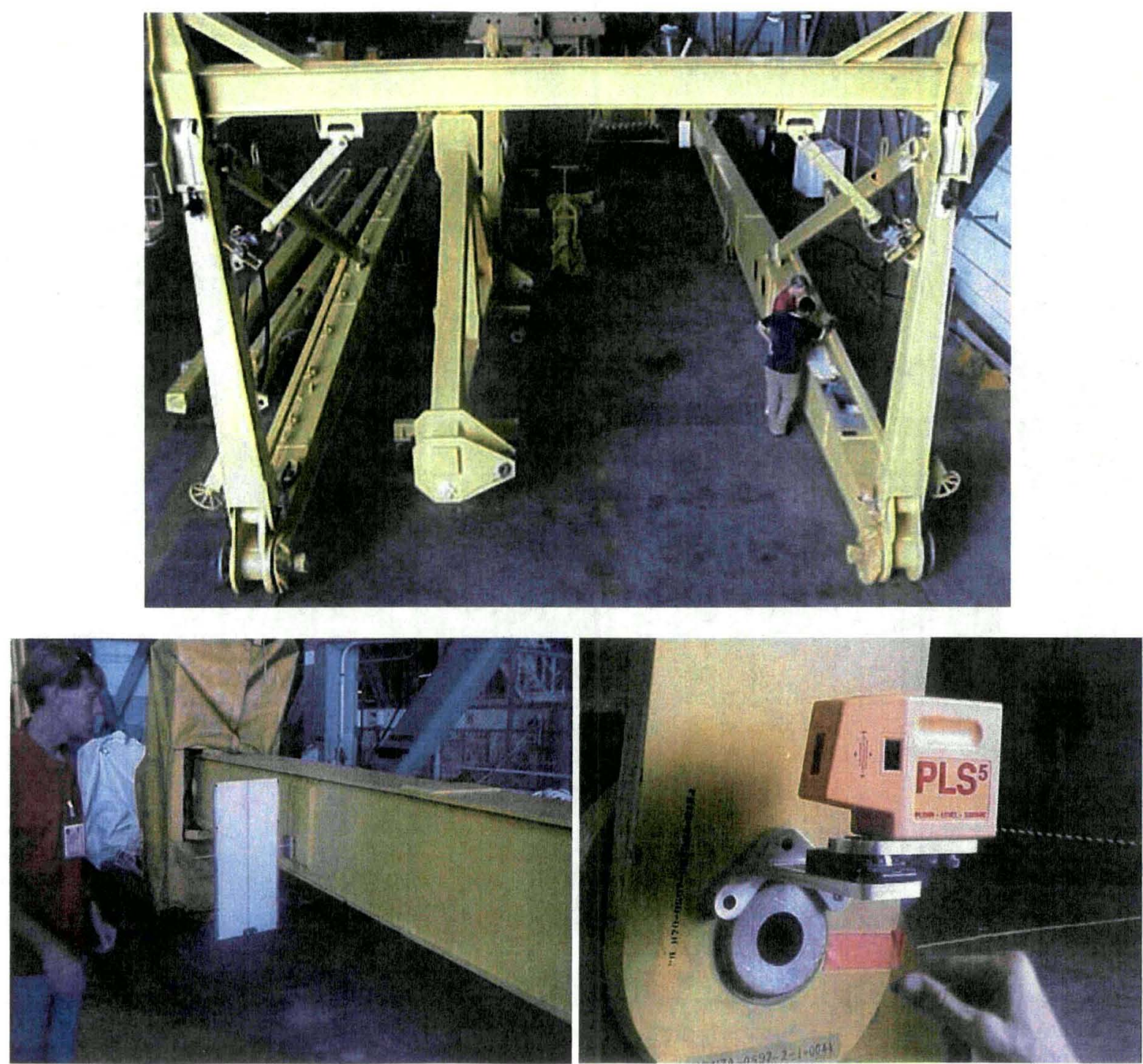

Figure 6. The Orbiter Sling Strain Sensor. The upper image shows the Orbiter sling, a piece of equipment used to pick the Orbiter up so it can be attached to the External Tank. Excessive strains can damage this structure so a laser based tool was constructed to provide a real-time visual indication of the strain.

Figure 6 shows the Orbiter sling, a large construct that is attached to the Orbiter allowing it to be lifted with a crane (the sling, attached to an Orbiter, is partially visible in the left hand side image of Fig. 1). The sling is clearly very rugged, but due to its length, excessive strains can be induced, especially in the corners. It has, on occasion, been damaged during handling and the KSC Applied Physics Laboratory was asked to provide advice on safely handling this important piece of ground support equipment. After some discussions it was determined that most of 
the damage was caused by shearing the structure. So a laser system was developed and delivered that provided a visual indication of this shear. An off-the-shelf laser unit was obtained that launched two beams at 90 degrees to each other. It was positioned to launch one beam across the front/short end of the sling and the other down its length to a white target (see Fig. 6 for the laser unit and distant target). As the sling is handled, shears cause the laser spot to move around on the target, but as long as the laser spot stays within a defined distance from center on the target the strains will not be excessive and will not damage the sling. This visual feedback of strain magnitude was accepted by the Orbiter processing team and put into use.

The final example in this section really isn't an alignment or positioning tool, but a remote measurement device. Yet, it was developed as a direct result of our earlier work and is included in this section. A problem experienced on the launch pad, typically with the External Tank, is the remote determination of the size of objects, a common example being damage in the ET foam. The foam acts as a cryogenic insulation and if it is damaged beyond specified dimensions it must be repaired, but most locations on the ET are not accessible, some areas being as far away as $\mathbf{8 0}$ feet from the nearest platform. Two of the authors (Stevenson and Rivera) realized that a solution to this problem would be to launch two or more parallel laser beams onto the External Tank. If the spacing between the beams was known, then the spot spacing would be known, and there would be a scaled reference image to measure against. The KSC Applied Physics Laboratory constructed two versions of such a device, one containing four red lasers with beams spaced by one inch and one containing two green lasers with beams spaced by 2 inches. Pictures of these two devices, both designed to attach to a camera so that a picture could be taken of the damage along with the laser beam spots, are shown in Fig. 7. Also shown in Fig. 7 are projected images of the respective laser beams on External Tank foam, one being used to scale bird scratches and the other an impact from a hail stone.
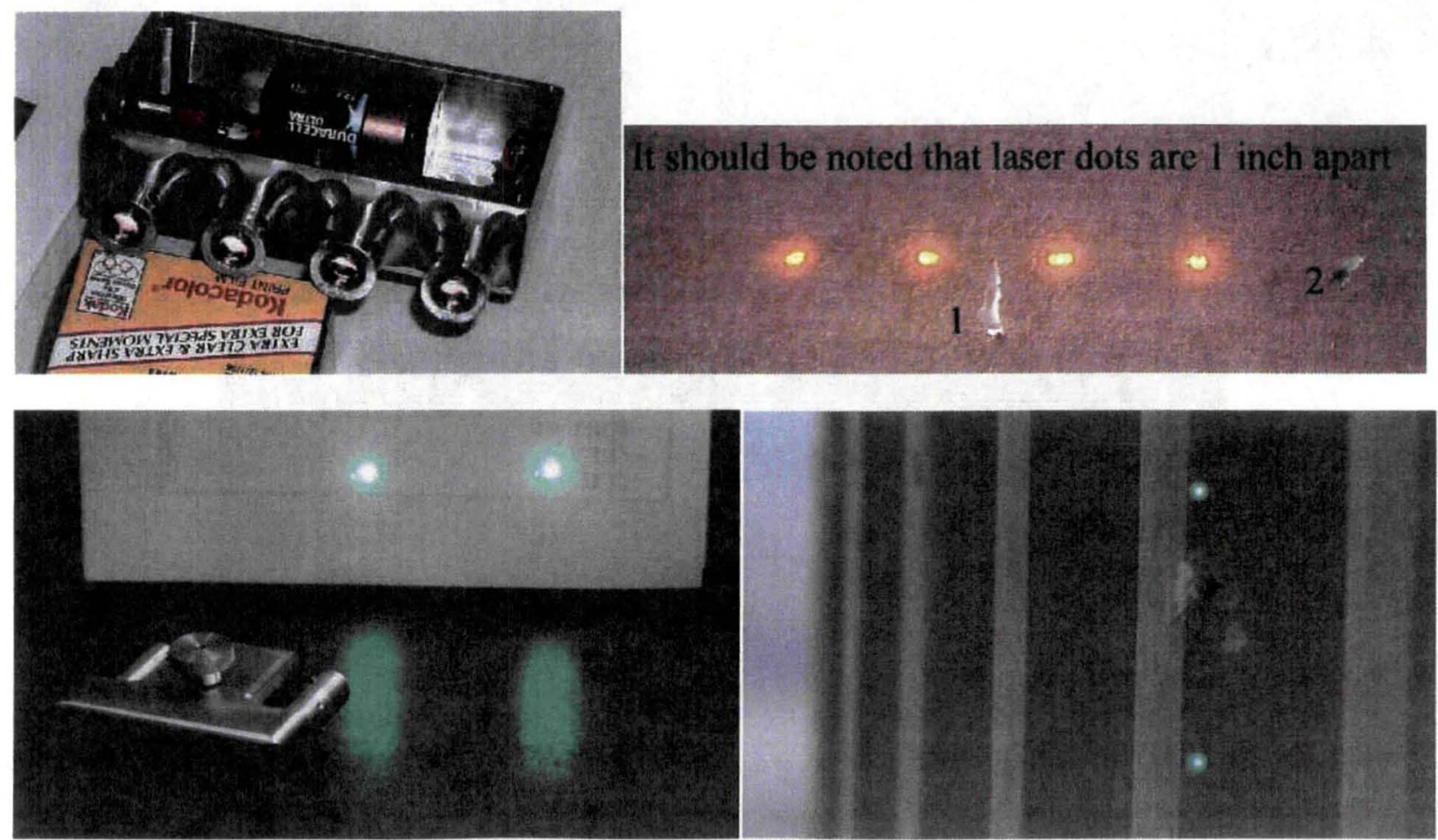

Figure 7. The Laser Scaling Device. This figure shows two versions of the laser scaling device, one with four red lasers spaced by one inch and one with two green lasers spaced by 2 inches. The right hand images shown the projected patterns being used to scale External Tank foam damage caused by bird damage or a hail stone impact.

This laser scaling device ${ }^{5,6}$ was advertised and commercial interest came from the forensics community. Crime scene photographers are required to produce scaled images of blood splatter, which can be difficult, requiring holding a ruler in the field of view without touching or hiding any of the blood. This can be especially onerous in rushed situations or when the blood is not easily accessible, for example on the ceiling. The scaling device allows a 
crime scene photographer to simply aim his camera and take pictures with the scaled image built into the picture. Consequently, NASA patented this concept, and signed a license for its manufacture and sale. Of particular note, the NAVY purchased several for use in Iraq and, we were informed, these simple devices helped to solve homicide cases. So a device originally meant to help measure bird scratches on the External Tank evolved into a tool to help resolve murder cases.

\section{B. Optical Systems}

While working on the positioning and alignment tools discussed in the previous section, the KSC Applied Physics Laboratory began to receive requests from Shuttle processing to develop inspection tools. The lab has expertise in optics and, combining that with the design and fabrication capability that now existed, many of these requests could be addressed. The work began with some relatively simple devices, but the complexity and significance grew as time passed.
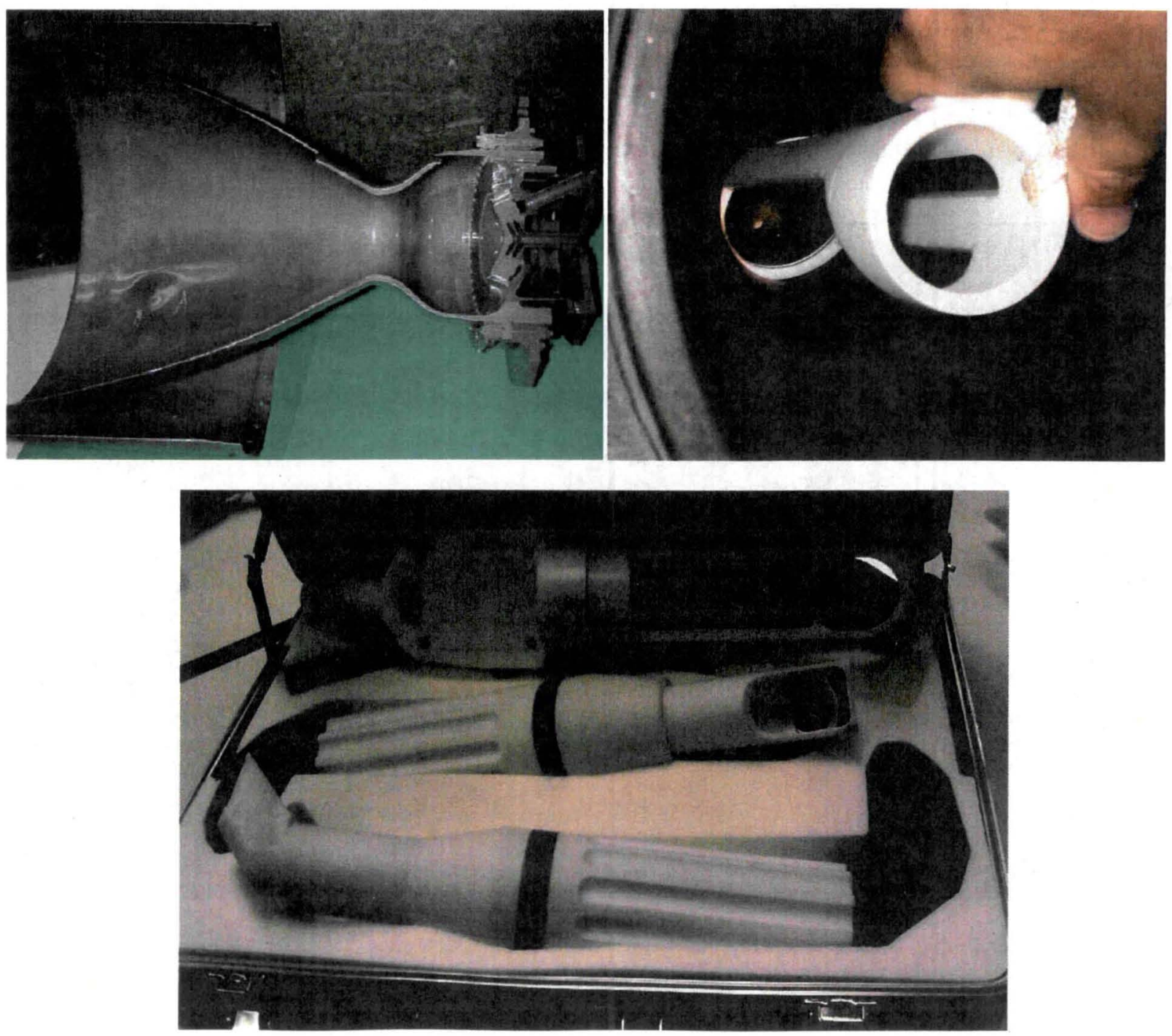

Figure 8. The RCS Nozzle Inspection Tools. The upper left image shows a cut away cross section of a Reaction Control System (RCS) Thruster. Thrusters, such as this, allow the Orbiter's position and orientation to be controlled while in orbit. Tools were designed and constructed, shown in the lower image, to allow these to be inspected. In the upper right one of these tools is shown imaging a small defect on the inner surface of the nozzle within the reaction chamber. 
While in orbit, the Orbiter's position and orientation are controlled by firing small thrusters, part of what is referred to as the Reaction Control System (RCS). These small rocket engines burn hypergols; liquid propellant (a form of hydrazine) and liquid oxidizer (nitrogen-tetroxide) that combust on contact with each other. The thruster, as seen in the cutaway image in Fig. 8, is composed of a manifold and inlets to direct and inject the hypergols into a firing or reaction chamber. This is followed by a shaped nozzle, directing the plume away from the Orbiter. Also, there are a large number of acoustic openings located around the firing chamber that admit nitrogen to help protect the nozzle. The nozzle itself is constructed from niobium (C-130) with a silicide based protective coating.

There are three primary goals in the inspection of these nozzles; to look for chips in the silicide coating, to determine if any of the hypergolic inlets are plugged, and to look for debris in the acoustic openings. Dental mirrors were being used to do this, but visibility was poor, viewing angles were not correct, and there was concern that the metal handles would damage the nozzle. The KSC Applied Physics Laboratory resolved these concerns by designing and delivering three custom inspection tools, each constructed specifically to meet one of the three different needs. Each tool was constructed with a Teflon body and plastic optics (both lenses and mirrors) to minimize the chance of damage and liberating debris. Each also had built in lighting (white light LEDs) to illuminate the inspection area. Figure 8 shows all three devices in the lower image. In the upper right image the coating inspection tool is shown being used to image a chip in the firing chamber of a nozzle on the Orbiter. The second tool, the hypergolic inlet inspection tool, has a built in lens to provide magnification and an angled mirror allowing the inspector to look into the inlets. The third tool, the acoustic opening inspection device, has a small periscope built into it allowing the inspector to look directly down the acoustic openings located along the outer edge of the thrusters firing chamber. All three tools were accepted into the hypergol processing area and were converted to shop aid status.

During the mid-1990s the KSC Applied Physics Laboratory was contacted by the group responsible for the Orbiter windows and this began a long and productive relationship, as demonstrated by the next four items. The six front Orbiter windows are each composed of three panes of fused silica, the inner two hold pressure while the outer one is sacrificial, meant to be replaced after acquiring significant damage from either handling on the ground or from micro-meteor impacts. The launch-commit criteria for the reuse of these sacrificial outer panes has evolved over the years, but is based on the depth of a single defect. A pane can continue to be used if it has hundreds of small defects, but the presence of one significant defect, one that might cause the window pane to crack under the stresses seen during launch or reentry, requires the replacement of the outer pane of silica. The difficulty is that this single defect can be very small, less than a thousandth of an inch deep, and to find all of the substantial defects on a window pane and then measure their depth is arduous and prone to error.

However, the windows inspection team noticed that if light were launched into the edge of an unmounted window that defects on the surface of the window would light up, making them easier to find. This, a very old idea, occurs because light is trapped between the window surfaces by total internal reflection when traveling at high angles relative to the surface. The light can only escape at defects (including dirt as shown in the right hand image in Fig. 9) until reaching the far edge of the window. So, the window inspection team requested a tool that could couple light into a mounted window, one where the edge was not accessible, such that the light would be trapped by total internal reflections and would allow the inspectors to be better able to find defects on the window surface.

The KSC Applied Physics Laboratory met this request by developing a small suction cup based device, shown in the lower left image of Fig. 9 that held a light pipe (not shown) pointed at the window at a steep angle. A small Plexiglas prism, painted black in the photo, was then used with water as a coupling agent, to launch light into the window at a steep angle, causing the light to be trapped. The image in the upper right of Fig. 9 shows a later, larger device, coupling light in this fashion into a block of Plexiglas: note how the edges of this transparent sheet are lit up and how fingerprints and scratches stand out. This device, called the surface light optimizing tool ${ }^{7}$ (SLOT) by Shuttle window inspectors, went through several design variations to improve the amount of light coupled into the window, to improve attachment strength to the window, and to improve the durability of the device. The final version is shown in the image in the lower right hand side of Fig. 9. This version has a strain relief for the light pipe (not shown), and two ports, one for a handheld pump that produces a partial vacuum to pull the unit firmly against the window surface and the other as a water inlet. This last version no longer has a solid prism, but fills a small volume with water through which the steeply directed light passes on its way to the window surface. Finally, this version includes a Plexiglas lens to help direct the light into the desired direction. SLOTs were readily accepted by 
the window inspection team and made locating defects easier. They were quickly accepted as shop aids and were used as a standard tool by the Shuttle Ground Processing Team.
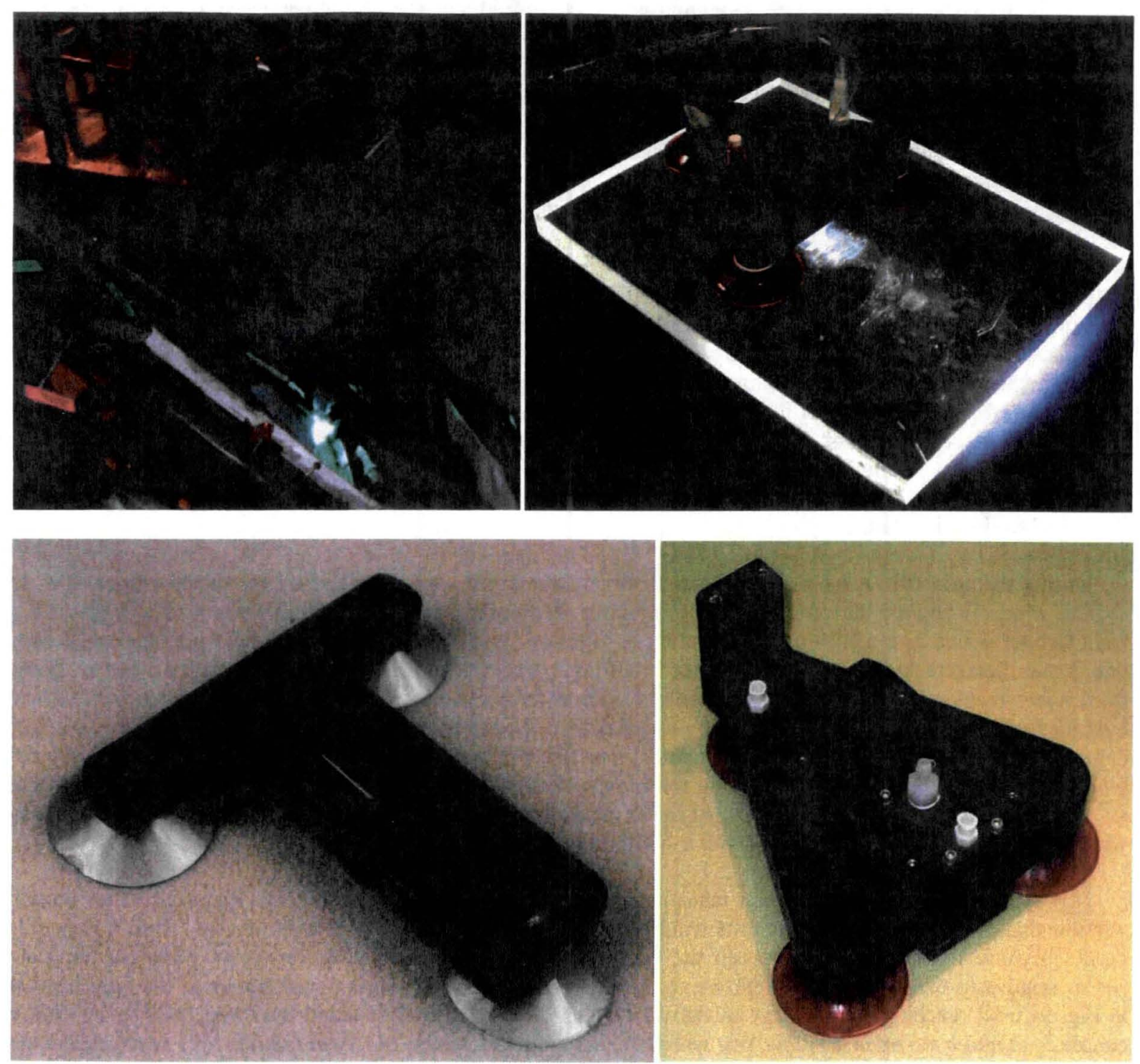

Figure 9. The Orbiter Window Surface Light Optimizing Tool (SLOT). Inspection of Orbiter Windows is difficult, due to access and the small size of the defects being sought. One early tool developed to make inspection easier was the surface light optimizing tool (SLOT). This optical device used a water coupled prism to launch light into a window at an angle steeper than the total internal reflection angle. As seen in the top right image, once light is launched in this fashion it becomes trapped and can only escape from the window at defects (including dirt and oils) or at the window edge. The final version of the SLOT is shown in the lower right image.

Later on, the Orbiter window inspection team requested a handheld camera system to help them quickly make a depth judgment on defects. A window pane might have hundreds of defects that turn up in inspection, but only a few need to be considered in detail as possible causes for replacing the window. Most defects do not pose a serious threat to the integrity of the windows, but a tool is needed to allow this decision to be made in a short time period. Historically, a refocus microscope was used for this purpose, but blind testing showed that potentially serious defects could be missed with this. So alternatives were sought. One option that the KSC Applied Physics Lab designed and delivered to help meet this need was a dual camera system where a wide field of view camera is bore- 
sighted with a short depth of field microscope. The wide field of view allows defects to be centered quickly in the field of view and then the short focal length microscope provides information on the depth of the defect. This device is shown in Fig. 10, where the focus adjust ring for the microscope is visible. This device was delivered late in the Shuttle Program, but was used by the window inspection team.

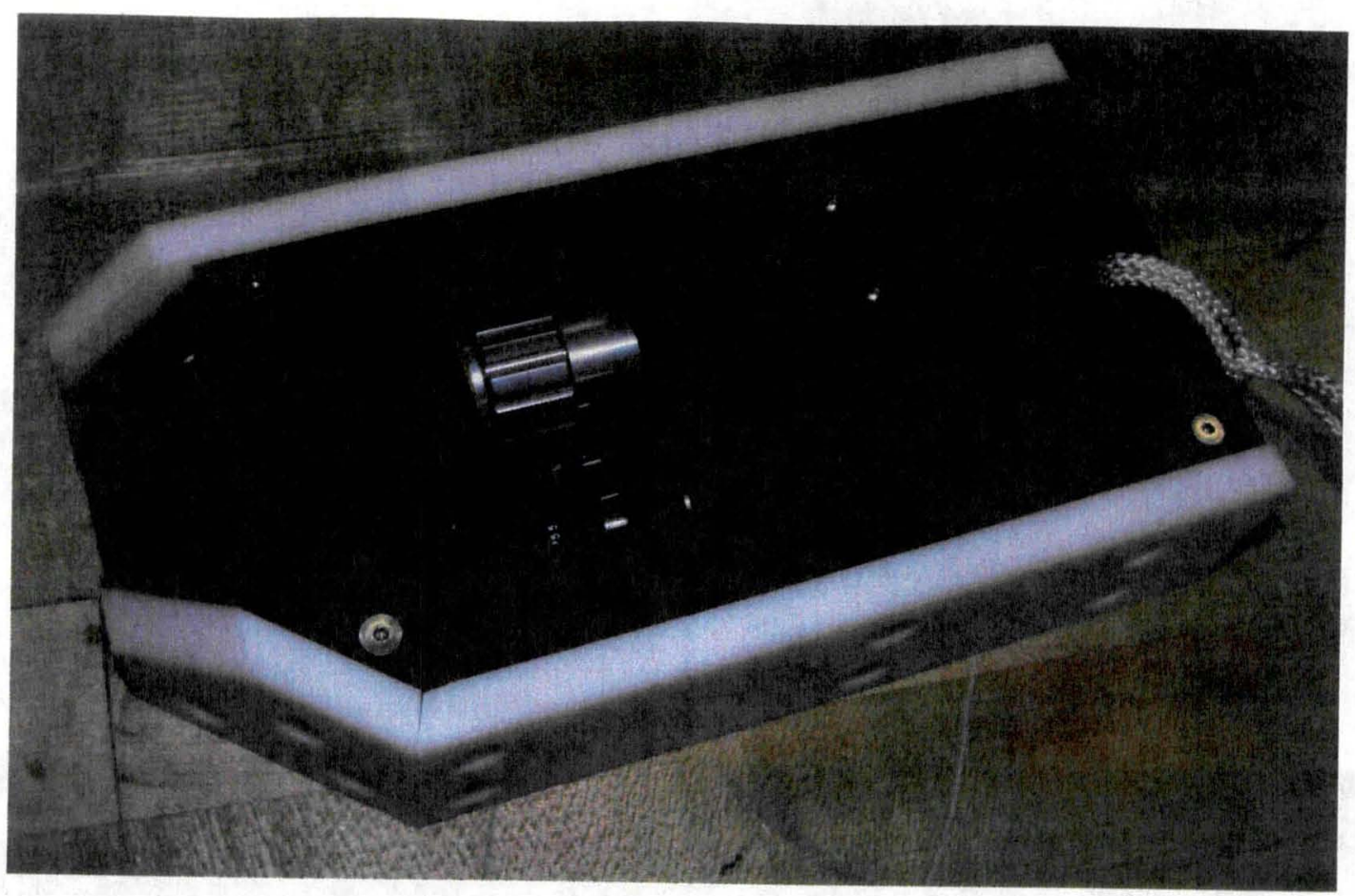

Figure 10. The Dual Camera Window Defect Imaging System. This is a picture of a dual camera system used to help determine if a defect on an Orbiter window needs more detailed measurements, or can be documented and ignored. It consists of a wide field of view camera, used to locate the defect, bore-sighted with a short depth of field microscope used to provide quick
depth information on the defect.

If a potentially significant defect is found on an Orbiter window pane, then more careful measurements are needed to determine if this might require pane replacement. Historically, this has been performed by taking mold impressions of suspect defects and then examining the molds using various optical tools. Transfer measurement is not ideal and introduces errors, but was necessary because no adequate device existed that could be placed onto the window pane itself and perform the measurement directly. Then, in the early 2000 time frame a potentially applicable technology became available that had been described in the literature in the 1990's, namely, chromatic confocal microscopy ${ }^{8}$. In this technique white light is sent through a custom lens that focuses different colors to different distances. A pen containing the lens is aimed at the glass surface and the color that reflects back up through the optical system determines the distance to the glass. STIL, a French company, was supplying systems for laboratory use, but also sold the individual pens, light sources, and spectrometers.

Shuttle window inspectors saw a STIL confocal microscope in operation at a conference and tried to fund the development of a portable version of this device through a U.S. distributor, but the cost was too high. So after some delay the KSC Applied Physics Laboratory offered to develop a portable version of this system ${ }^{9}$, the final embodiment of which is shown in the top left image of Fig. 11. This device uses suction cups to attach itself to the Orbiter window pane and contains a small camera and display allowing the user to properly position the device near the desired defect. White light comes into the device along the orange fiber-optic guide and enters the chromatic lens assembly in the small black tubular pen. Stepper motors driving translation stages are used to raster scan the lens assembly over the defect, repeatedly taking depth measurements to generate a 3-D topographic map. The optical spot size is less than 1 mil and the depth resolution is better than 30 micro-inches (about 1 micron) allowing high resolution mapping. The image in the upper right hand side of Fig. 11 is an optical picture of a moderately large 
window defect (about $0.3 \mathrm{~mm}$ largest dimension). The lower image in Fig. 11 is the output generated by the defect inspection device looking at this defect, including the desired 3-D map, various plots, and defect parameters.
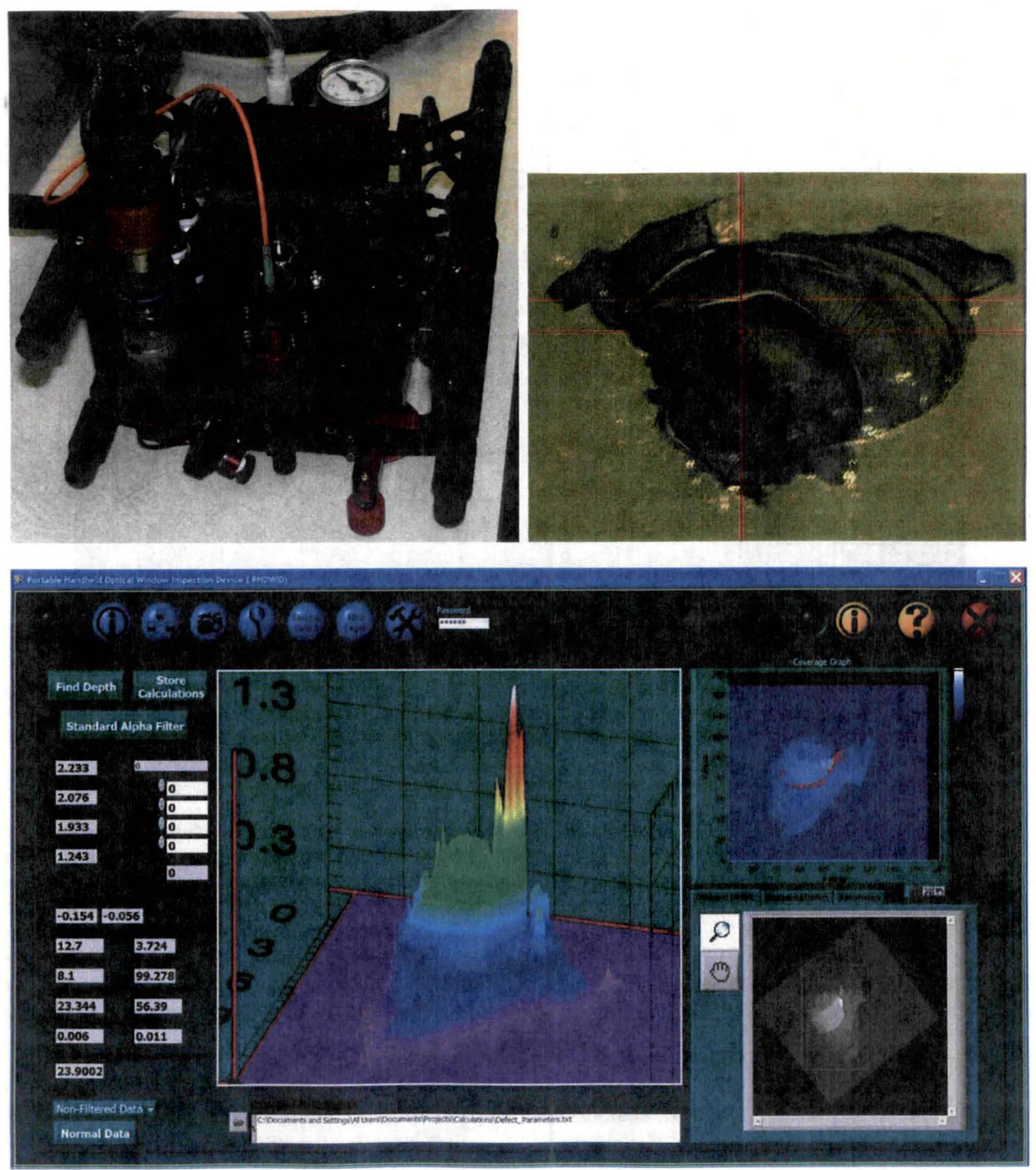

Figure 11. The Portable Window Defect Inspection Device. The upper left image shows a window inspection device developed to provide detailed 3-D maps of defects on the Orbiter windows. The upper right shows a visual image of a relatively large window defect $(0.3 \mathrm{~mm}$ largest diameter) and the lower image shows the 3-D map, plots, and output generated by the inspection device from this defect. 
The window defect inspection device was, sadly, not used by the Shuttle Program, due to delays in certification. Testing showed it to be a preferable replacement to the use of mold impressions, but as the Shuttle Program approached its end it became difficult to introduce new equipment. The current plan is to deliver two of these units to hypervelocity impact researchers at the Johnson Space Center.

In May 2006 the launch commit criteria for Orbiter window panes became more stringent, requiring that a larger number of defects be located and measured. This posed a serious challenge to the Space Shuttle window inspection team and they began removing window panes from the Orbiter so that they could be qualified in an off-line lab. In the mean-time a rush project was begun within the KSC Applied Physics Laboratory to construct a scanning instrument that could be directly mounted to an Orbiter that would locate and order by size all of the significant defects on a window pane. During the fall of 2006 a prototype was tested on an Orbiter and it worked well enough that design and construction began on a deliverable version. This window inspection system consisted of a camera head that contained a microscope, a stress imaging camera, and a pen that could be lowered onto the window to circle and number selected defects. The stress imaging camera operated by launching polarized light through the window, bouncing it off of the back surface so that it passed through the area being inspected and imaging the changes in polarization, taking advantage of the relationship between stress and optical propagation in glass (see the right hand image in Fig. 12). This approach worked well and the final version of the system, shown in the left hand image in Fig. 12, was tested on every Orbiter. But then, the launch commit criteria was eased, removing the need for off-line laboratory testing of the windows and allowing the window inspectors to return to their earlier methods and removing the need for the window inspection system. Although this system was not implemented into operation, it has been included in this summary as an example of the technical development level achieved by the KSC APL in its support of the Shuttle Ground Processing Team.
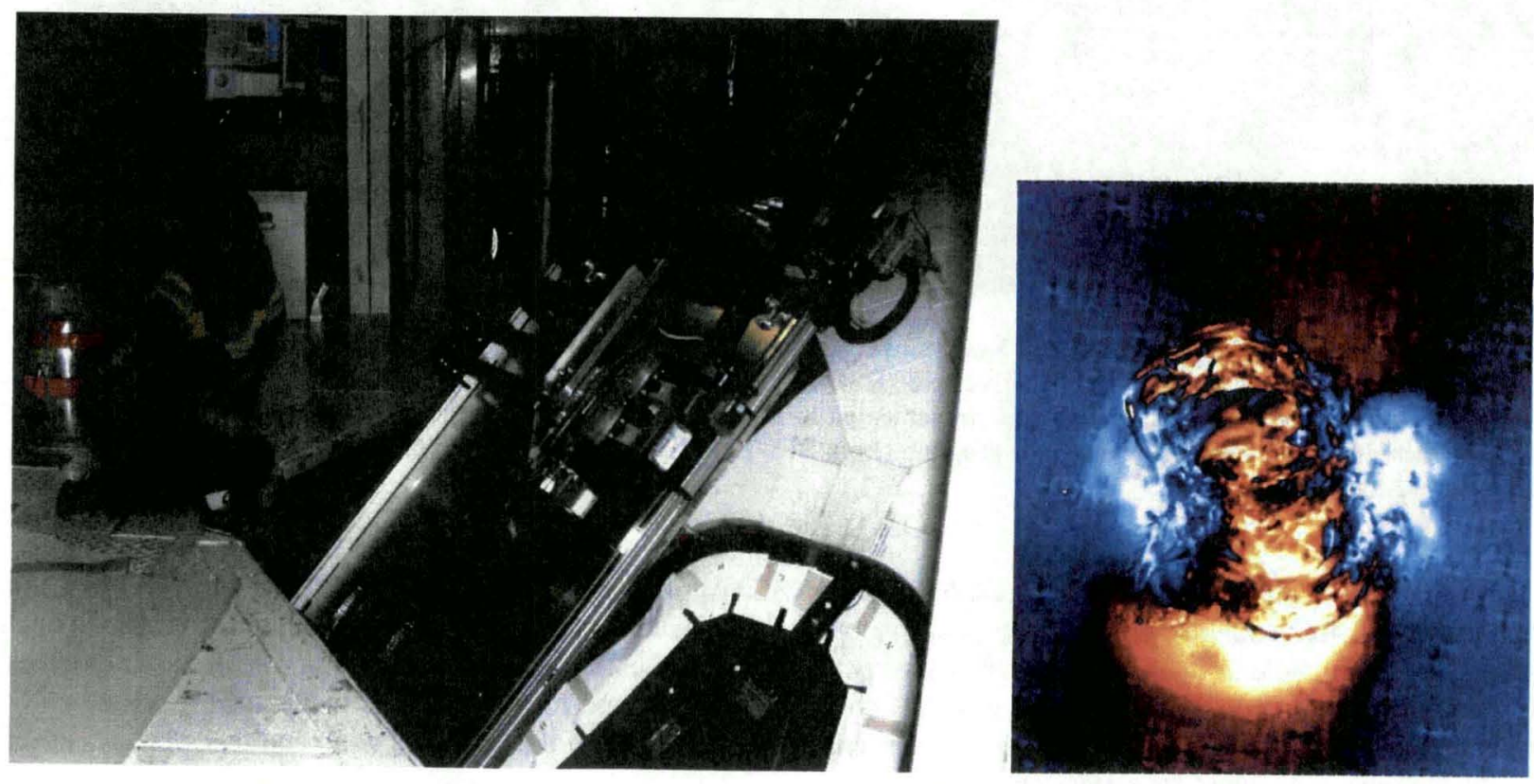

Figure 12. The Orbiter Window Inspection System. To the left is shown an Orbiter Window Inspection System where a microscope and a stress imaging camera are raster scanned over an Orbiter window in an OPF. To the right is an output image from this system showing a color coded stress field associated with a defect.

Shortly after ignition of STS-114 (July 2005), as the Space Shuttle was clearing the launch pad, the External Tank impacted a vulture. The Space Shuttle was already moving fast enough to kill the bird which subsequently fell into the SRB rocket plume. This was an undesirable event for two reasons, impacts to certain portions of the vehicle can be damaging, possibly causing a launch abort, and killing wildlife within the Merritt Island National Wildlife Refuge (within which the Kennedy Space Center is located) is prohibited. So a team was formed, which included the KSC Applied Physics Laboratory, to find ways to prevent another vulture impact. Many vultures were living near the launch pad and using the updrafts to glide above the Space Shuttle (see the left hand image in Fig. 13), but most 
standard bird removal concepts could not be used either because of issues with the Space Shuttle or because it might be injurious to the birds.

Eventually it was realized that the vulture problem partially stemmed from a population increase resulting from road-kill. Wildlife impacts by vehicles driving on KSC roadways were supplying a plentiful food source for the vultures resulting in an increase in their numbers. So a partial solution to the problem was to simply hire someone to pick-up and dispose of road-kill before the vultures could get to it. This alone led to a significant reduction in the local vulture population and fewer were observed gliding over the launch pads. In addition to this, two vulture tracking systems were installed and used to support every Shuttle launch from STS-121 to the end of the program. The first was a radar system developed by JSC personnel and the other was a multi-camera system developed and operated by the KSC Applied Physics Laboratory. The camera system used three existing cameras to monitor the region over the launch pad from a few hours before launch until launch itself. Three operators, each monitoring a camera, then assisted a Lab View program in using triangulation to track vultures in the 3-D space over the pad. The imagery (see the right hand side of Fig. 13) was sent to the launch director providing information on the likelihood of the vehicle hitting a vulture on launch.

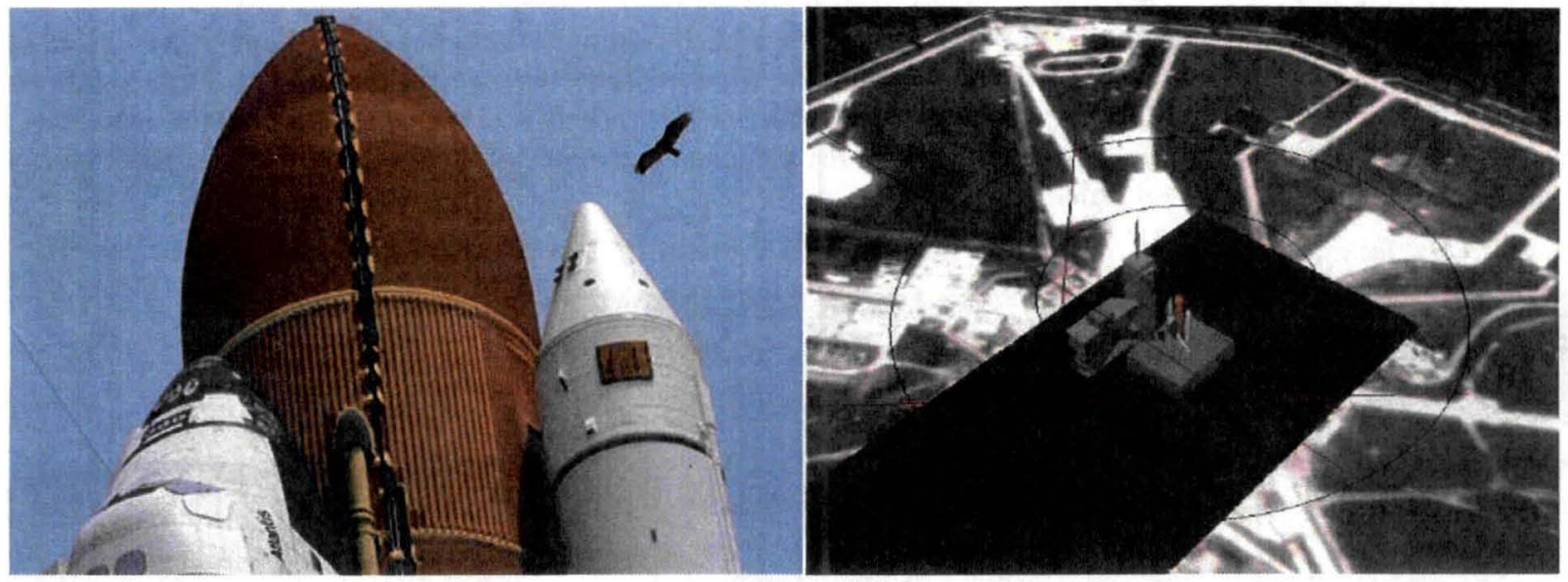

Figure 13. The Pad Vulture Monitoring System. The Space Shuttle once hit a vulture during take-off. This is potentially dangerous to the Vehicle and deadly to the vulture (KSC is part of a wildlife refuge so this is not an acceptable situation). Consequently, a vulture tracking system was implemented to track vultures flying over the pad and has been in operation for every launch during the last five years of the program (from STS-121, 7/2006). A typical output from this system is shown in the image to the right.

During the Columbia accident investigation, a KSC Applied Physics Laboratory Contractor, John Lane, developed the mathematics to track the position of the ET foam debris relative to the Orbiter from the launch imagery ${ }^{10}$. Being able to view the foam from two perspectives and using known points on the Orbiter as references, Lane developed a method for establishing a 3-D coordinate system and locating objects within this. Consequently, he was able to deduce the speed at which the foam hit the leading edge of the Orbiter's wing and this work was used by the Columbia Accident Investigation Board.

After Lane did this, it was recognized that the same algorithm could be used to track launch debris, as long as the debris could be seen by more than one camera ${ }^{11,12}$. The Space Shuttle Program funded the development of this software and included it in its photogrammetry tools to help track the trajectory and determine the final locations of objects seen in launch videos. Then, a couple of years later, a forensics company approached the KSC Applied Physics Laboratory and asked if there was a method where a crime scene photographer could take pictures with a standard camera, and later on be able to determine 3-D location and distance information. Dr. Lane recognized that this was a direct extension of his Shuttle software development and was possible as long as stable reference points were included in the images. So he had a reference cube constructed (see Fig. 14) and demonstrated that if pictures were taken of a scene from two or more perspectives with the reference cube in the pictures, that any 2-D images in two or more pictures could be converted to 3-D information. This simple system, requiring only a reference object, 
such as the cube, and a standard camera, replaces the need for an expensive 3-D scanning system. It has been used for several measurements within the NASA community, typically to measure sensitive space hardware components that cannot be touched or moved. In addition, this system was recently taken to Haiti by a civil engineering professor to use to measure displacements in structures caused by the 2010 earthquake.
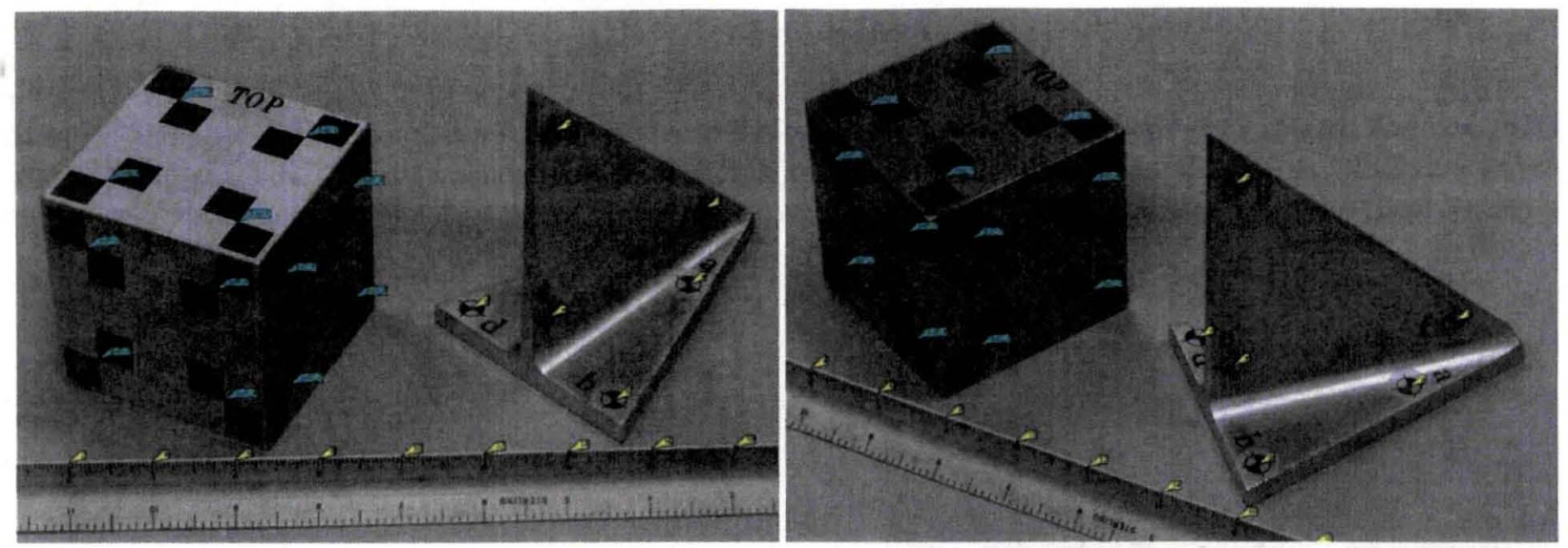

Figure 14. Three Dimensional Object Tracking using Multiple Images with Reference Points. Using a mathematical algorithm developed to track foam debris in Columbia imagery, a software package was generated to track debris at the launch pad. This software was later modified to create a system where 3-D location information could be obtained from 2-D imagery, an example of which is shown in this figure.

\section{Water/Moisture/Ice Systems}

Water, in its various forms, has caused problems for the Space Shuttle Program. Liquid water can be absorbed into the tiles and blankets that make up the thermal protection system, ice can form on the External Tank during fueling operations and become source of debris that could fall onto the Orbiter, and hailstones can hit the External Tank causing foam damage. These issues have all been brought to the attention of the KSC Applied Physics Laboratory and this section of the paper discusses the solutions and the hardware developed to address them.

The Orbiter has over 20,000 tiles forming a thermal protection system. These tiles are composed of nearly pure silica fibers that form a rigid material with extremely low thermal conductivity. They are lightweight, only 7 percent of their volume is glass with the rest being air (or vacuum while in orbit), and coated with a thin layer of typically black glass in order to raise their emissivity. Overall, they are an ideal material for this purpose except that they have great affinity to water. A typical acreage tile (about 6 inches by 6 inches by $2 \frac{1}{2}$ inches in size) contains about 40 million meters of fiber with a net surface area of 180 square meters. This means that the capillary forces within a Shuttle tile are large, causing it to act as a state-of-the-art sponge, pulling water into it and not easily releasing it. So in practice every tile on the Orbiter is injected with water proofing, through a small water-proofing hole, before launch so that the vehicle can be taken to the launch pad and not soak up water from rain. If the tiles were to soak up water it would add excessive weight to the vehicle (a typical tile can hold a kilogram of water) and would cause stress on the tile due to boiling during ascent or due to freezing while in orbit.

The problem is that the aerodynamic heat generated during reentry burns the waterproofing out of the tiles. This isn't serious when landing occurs at KSC because the Orbiter is brought indoors quickly, but when it lands on the west coast it can be exposed to substantial water before returning to KSC. In 2001 Atlantis landed at Edwards Air Force Base and was exposed to large amounts of rain, saturating essentially every tile on the underside of the Orbiter. Shuttle Ground Processing tried to remove this water with heat lamps, but after weeks of trying water was still present, resulting in a delay in processing and subsequently in launch. A wet tile team was formed and the KSC Applied Physics Laboratory was asked to help find a better way to remove water from the Orbiter's tiles. 
Laboratory personnel soon realized that the best way to remove tile water was to apply a partial vacuum to the water-proofing hole ${ }^{13}$. It was demonstrated that most of the water in a tile could be suctioned out within an hour and a half through this small opening. A successful demonstration was given to the tile team and it was agreed that a field version should be designed and fabricated that used the OPF's built-in vacuum cleaning system. As a result, four systems were constructed, each composed of a water collection tank from which five large lines emerged, each of those breaking into 5 smaller lines that ended in suction cups. So, all told the four systems could dry 100 tiles at a time using the OPF's vacuum system.

Then, in August of 2005, STS-114's (return to flight mission) Orbiter, Discovery, landed at Edwards Air Force Base, and was subsequently soaked by rain. The four Water Extraction Tools (WET) were quickly fielded and used to dry every tile on the Orbiter. Figure 15 shows two of the units located underneath the Orbiter with 50 suction cups attached to tiles. It also shows a technician attaching suction cups to wet tiles allowing the vacuum system to extract their water.

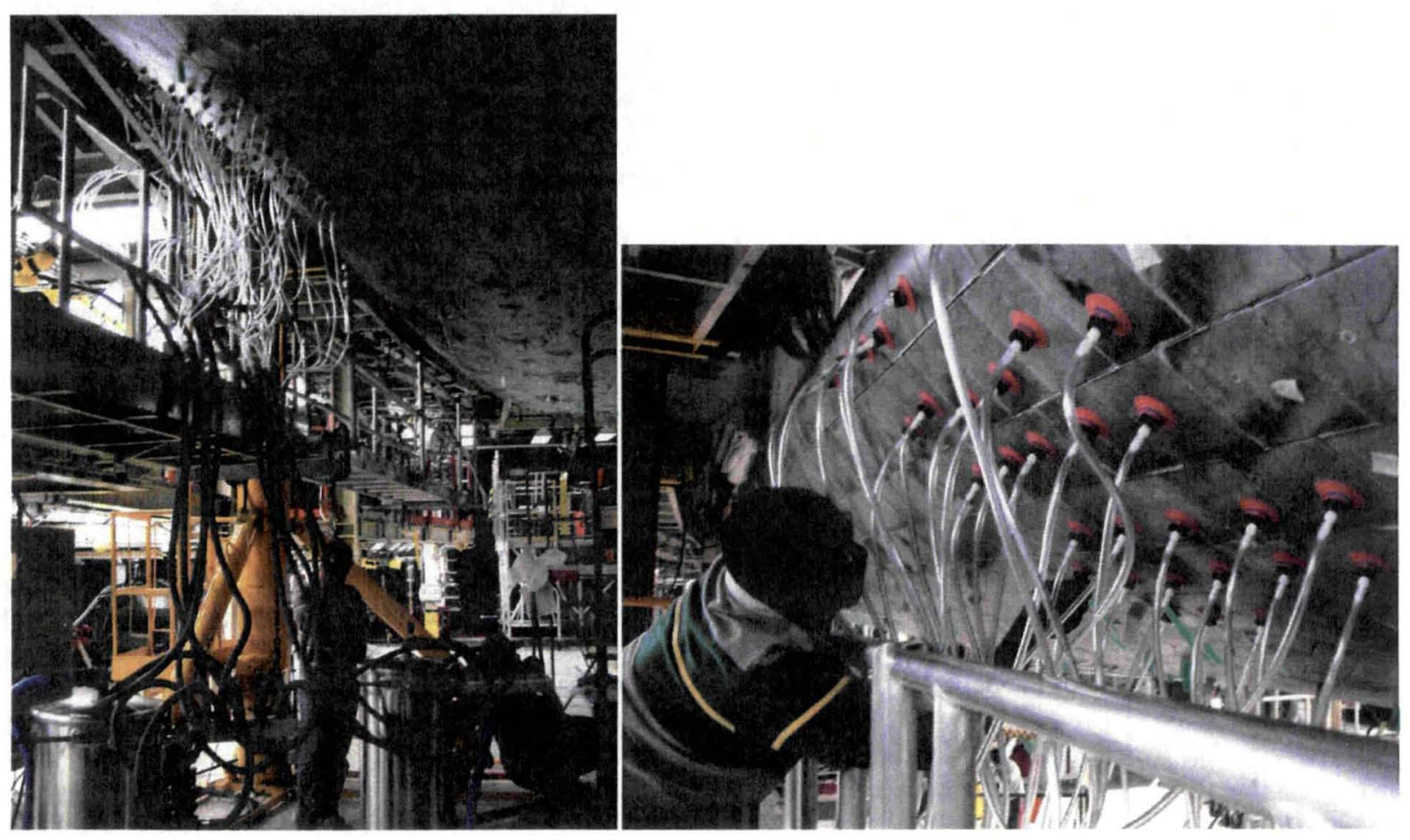

Figure 15. The Water Extraction Tool. A Water Extraction Tool (WET) was developed and delivered to the Shuttle Ground Processing Team to vacuum water out of wet Orbiter tiles.

Then, after using the WET, the Shuttle Processing Team wanted to know if a tile was indeed dry; had all the water been removed. To help determine this two different sensors were developed. The first was designed specifically for tiles and operated by drawing a small air sample from a tile, again using the water proofing hole, and measuring the humidity of this air ${ }^{14}$. The idea was that since the air within a tile cannot recirculate easily with air outside of the tile, that small amounts of water would saturate the air within the tile. So if the air in the tile was wet, it would signify the presence of water. This device was well received by the processing team and was soon classified as a shop aid. An image of it is shown on the left hand side of Fig. 16. It consists of a battery operated pump that allows air to be drawn through the red suction cup and then through a humidity sensor. The second device was a capacitance sensor, taking advantage of the high dielectric constant of water and operating in a similar fashion to standard stud finder. Originally this device was also designed for use on tiles, but the humidity sensor soon filled that need. Instead, the capacitance sensor was accepted as a tool to determine if the blankets used on other surfaces of the Orbiter contained water. This tool is shown in the right hand image of Fig. 16. 

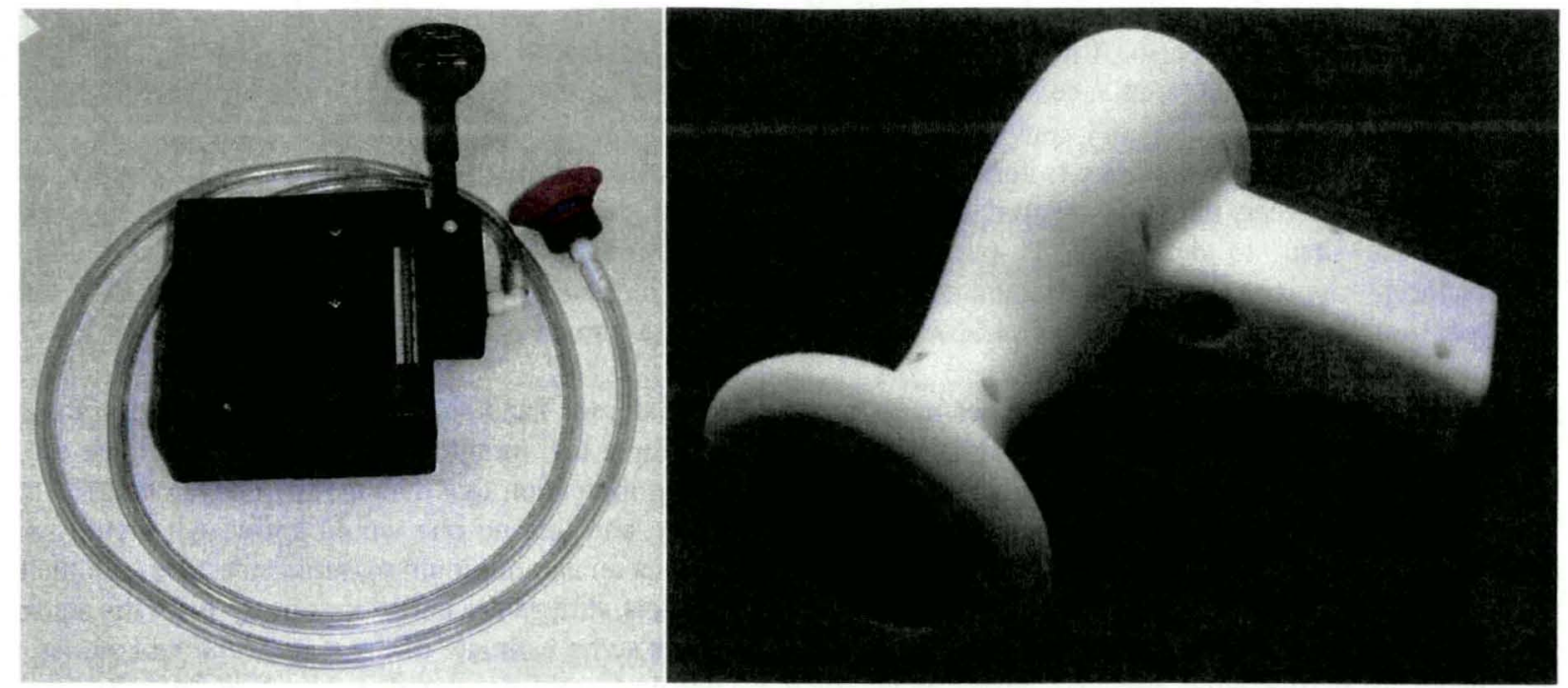

Figure 16. Water/Moisture Detection Devices. These are two different moisture detectors delivered to the Orbiter Processing Team. The left hand image shows a device that monitors the humidity of air samples, allowing the detection of water in Orbiter tiles. The right hand image shows a capacitance based device that was used to help find moisture in blankets.
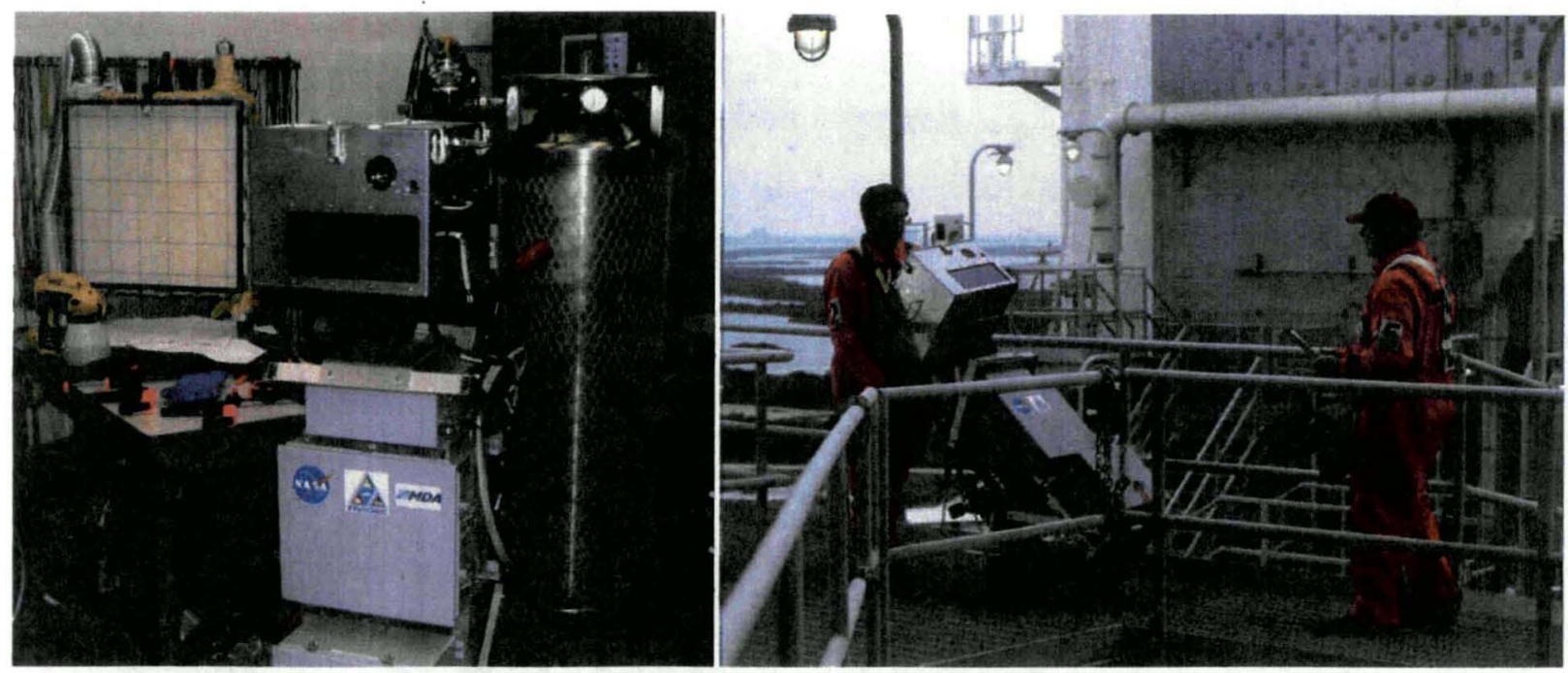

Figure 17. Certification of an Ice Thickness Measurement Camera. An outside company constructed an ice camera for use by the Shuttle Ice Team in measuring the thickness of ice on the External Tank during fueling. The KSC Applied Physics Laboratory developed custom instrumentation to determine the capabilities of this camera.

Ice can form on the External Tank during fueling and there is a concern that this ice could fall onto the Orbiter during ascent and cause damage. Consequently, there is a launch-commit-criterion that limits the amount of allowable ice on various surfaces of the External Tank, in some locations no ice is allowed and in others up to $1 / 16^{\text {th }}$ of an inch is permitted. The problem is that these areas are not directly accessible and trying to judge the thickness or presence of ice on a surface up to 80 feet away is difficult. An ice team goes to the pad to assess the situation before each launch, but it was recognized by Shuttle Processing that they needed help. So, a request went out for ice imaging/thickness measurement systems and one outside company claimed to be able to make such a device ${ }^{15}$. They were given a contract under which they designed and fabricated an ice camera that they said would meet the needs of the Shuttle Program. 
The ice camera was delivered to the Shuttle Program in late 2005 and the KSC Applied Physics Laboratory was asked to determine if it could indeed measure ice thickness to better than $1 / 16^{\text {th }}$ inch accuracy at the required operational distances. To perform this test a custom liquid nitrogen Dewar was constructed onto which a panel of External Tank foam could be attached (see the left hand image in Fig. 17). When this Dewar was filled with liquid nitrogen the foam would drop to below freezing and then a paint sprayer filled with water could be used to spray droplets onto the foam, causing ice to form. Varying parameters allowed the growth of clear ice, light snow, or any ice density in between that was desired, to known thicknesses. The camera could then be tested against this accessible ice sheet to determine its performance. After significant effort - and many interactions with the manufacturer - the ice camera was deemed capable of accurately measuring small thicknesses of ice. It was used, as a support tool, by the ice team, on several Space Shuttle launches as shown in the right-hand image of Fig. 17.

There have been cases where hail stones have impacted the External Tank and have caused damage to its foam. In 2002 the KSC Applied Physics Laboratory was asked to develop a hail monitor ${ }^{16,17}$ that could determine the size of hail stones falling around the launch pad in order to provide an indication that damage may have occurred to the ET. There was a concern that hail might fall, damaging the tank, and that no one would know. After study a hail sensor was fabricated based on monitoring the impact of hail stones on an aluminum pyramid structure (see the right hand image in Fig. 18). This design separates rain from hail impacts, directs the hail stones away from the sensor so that secondary impacts from bouncing are minimized, and allows some estimate of the size of the hail stones. The completed system was solar powered and included an aluminum wrapped foam panel as a back-up record of the hail events. Three of these were constructed and placed at the pad whenever a Shuttle was present. Then, in 2007 an intense hail storm passed over the launch pad, significantly damaging the ET (see the left hand image in Fig. 18). The hail monitors provided information on the stone sizes and relative number of impacts to the Space Shuttle Vehicle. They continued to be used at the launch pad until the end of the Space Shuttle Program.
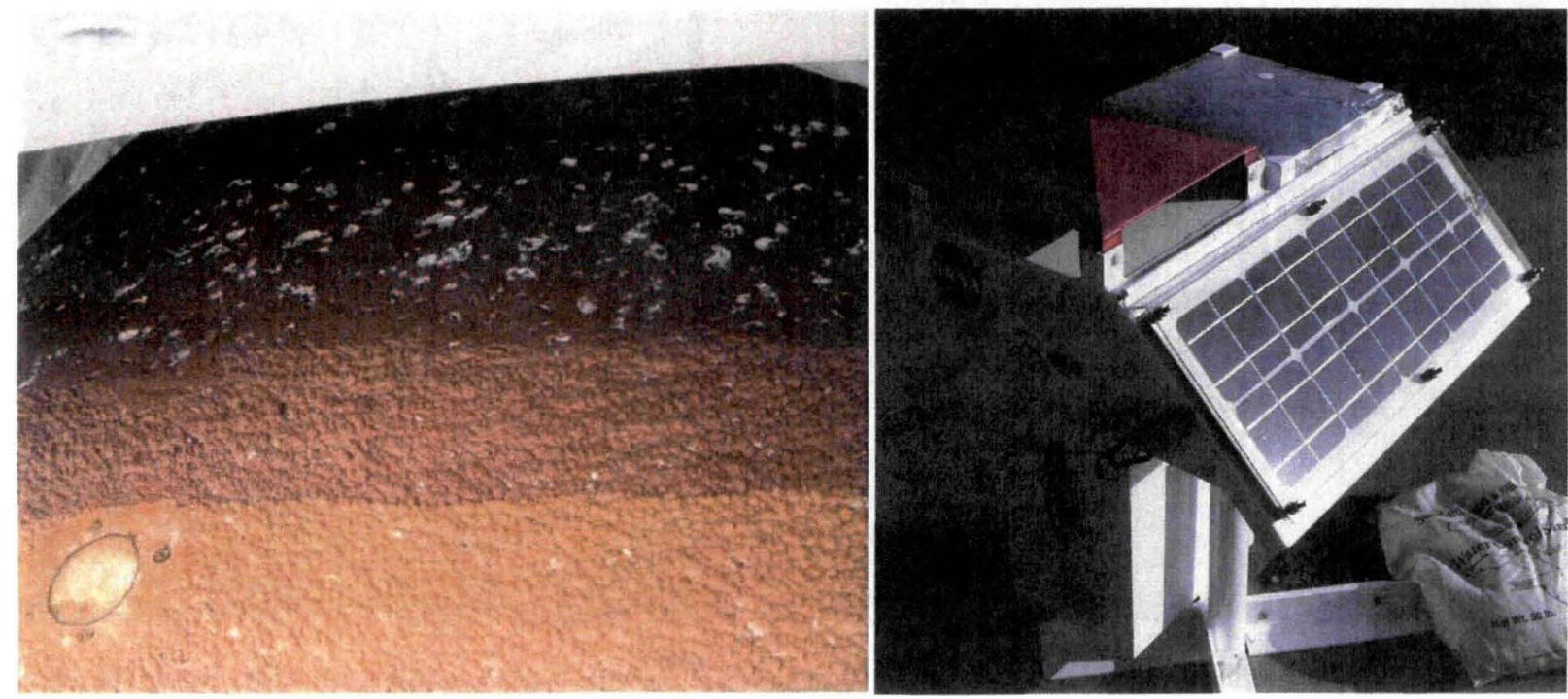

Figure 18. A HailStone Monitoring System. On the left side is an image of hail induced damage on the External Tank foam. On the right hand side is a hail monitor developed by the KSC Applied Physics Laboratory and used to support Shuttle launches.

\section{Sensors}

This section describes requests that have come to the KSC APL to provide either sensor or measurement support. These requests represent somewhat diverse topics, but all reflect a need to measure something and most have resulted in long-term relationships with various portions of the Space Shuttle Ground Processing Team at KSC. The five topics discussed in this section have all made significant impacts, but have also resulted in several patents and publications. They reflect the innovation that can occur when working closely with an operational space-port. It's 
also interesting to note that several of the requests described in this section concern some aspect of hydrogen, reflecting the ubiquitous nature of this Shuttle fuel at the Kennedy Space Center.

Hydrogen fires are invisible to the eye in daylight conditions and represent a serious potential danger to both the pad and its personnel, so it is important that these fires, when present, be detected quickly. Even though hydrogen fires do not emit substantial amounts of visible radiation, they emit large amounts of infrared and a small amount of ultraviolet, both of which are used as the basis for commercial hydrogen fire detectors. There is more infrared to detect, but there are more possible interfering sources that could yield a false alarm, so ultraviolet detectors are used at the launch pads. These fire detectors only alarm when radiation with wavelengths between 180 and 250 nanometers reaches the detector. Since the ozone layer blocks radiation from the sun below $300 \mathrm{~nm}$, the world is normally dark in this band and only fires and few other sources can generate this short wave radiation needed to alarm the fire detectors. The problem is that the detectors age badly, losing their sensitivity and falling out of calibration quickly, so it is required that their performance be checked before every launch. To accomplish this, the Shuttle Ground Processing team obtained a commercial UV source, but it was very bright, causing even minimally performing detectors to alarm and in some cases caused the detectors to latch up, continually alarming even after the removal of the source. Clearly a better device was needed and the KSC APL was approached with this need.

The KSC Applied Physics Laboratory developed a handheld ultraviolet hydrogen fire simulator ${ }^{18,19}$ to solve this problem. The device, shown in the left hand side of Fig. 19, emits known amounts of ultraviolet radiation in the detection band of the fire detector, allowing not only the operation of the detectors to be verified, but that the detectors are operating within reasonable design limits. This device has an internal calibration feature allowing microprocessor control of the amount of ultraviolet emission, providing confidence that it is emitting the correct amount of radiation. As time went on and the device gained pedigree it was written into the Shuttle Launch processing procedures and is now the primary tool used to certify the performance of the pad fire detection system. There was sufficient commercial interest in the handheld hydrogen fire simulator that it was patented, but it was never produced as an off-the-shelf item.

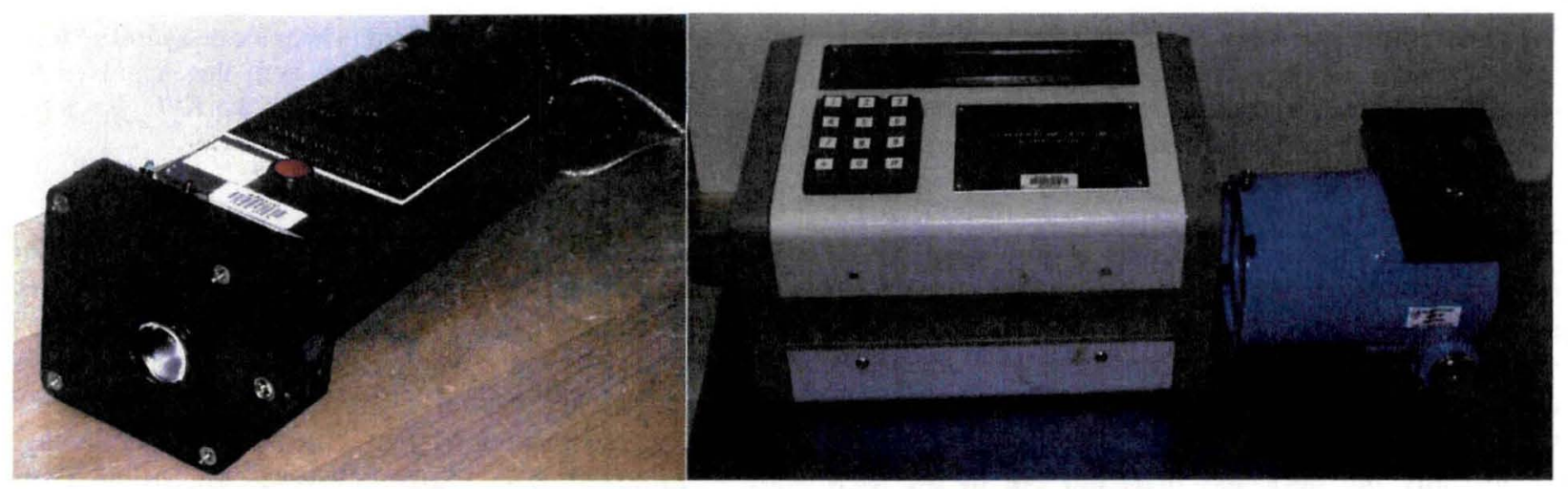

Figure 19. An Ultraviolet Hydrogen Fire Simulator and Fire Detector Calibration Tool. Hydrogen fire detection is performed at the launch pads using ultraviolet detectors. The image to the left shows an ultraviolet flashlight that produces a known amount of ultraviolet radiation, allowing it to be used as a portable hydrogen fire simulator to certify the fire detection system. The image to the right shows a table-top device designed to test the ultraviolet fire detectors, the blue cylindrical object, in a laboratory environment.

After this the Shuttle team asked the KSC Applied Physics Laboratory if a tabletop certification, or possibly even calibration, device could be constructed for the ultraviolet fire detectors. Safety had restricted the use of actual hydrogen fires and Shuttle operations found it difficult to perform acceptance testing of the fire detectors. What they wanted was a device that would make it appear that a standard flame was being slowly moved away from a fire detector so they would know at what distance the fire detector stopped alarming. The KSC APL fabricated a relatively sophisticated device $e^{20,21}$, shown in the right hand image of Fig. 19, that performed this function. The device produced a uniform and known amount of adjustable ultraviolet radiation while monitoring the fire detector. Again, there was commercial interest in this device, such that it was patented, but it was never put into production. 
In the early 1990's hydrogen leaks caused the entire Shuttle fleet to be grounded until their source could be found. During this period the KSC Applied Physics Laboratory developed a small ultrasonic leak detector to help with the search. This device was placed into an Orbiter aft compartment during a leak test to listen for the ultrasonic emission associated with a pressurized gas leak. It was not the key technology that found the leak, but it worked well enough that Shuttle operations asked for further development in this area. They conceded that they owned a commercial ultrasonic leak detector, but its sensitivity and directionality were not sufficient enough to be useful. So the lab began work in this area and came up with a parabolic receiving horn that improved the sensitivity of a standard ultrasonic leak detector by about a factor of six and limited the directionality to a narrow beam, providing quick location of a leak ${ }^{22,23}$. Several complete ultrasonic leak detectors using this horn concept, shown in the left hand image of Fig. 20, were fabricated and delivered. Then, an outside company showed interest in this work, so the concept was patented and followed by a commercial device, shown in the right hand image of Fig. 20. The positive reception from both Shuttle and our outside commercial partner led to the subsequent development of an ultrasonic camera, a device with a $12 \times 12$ grid of ultrasonic sensors and a large reflecting dish that acted as a lens. This device allowed ultrasonic emission to be imaged and it was patented ${ }^{24,25}$, but it was never put into production.
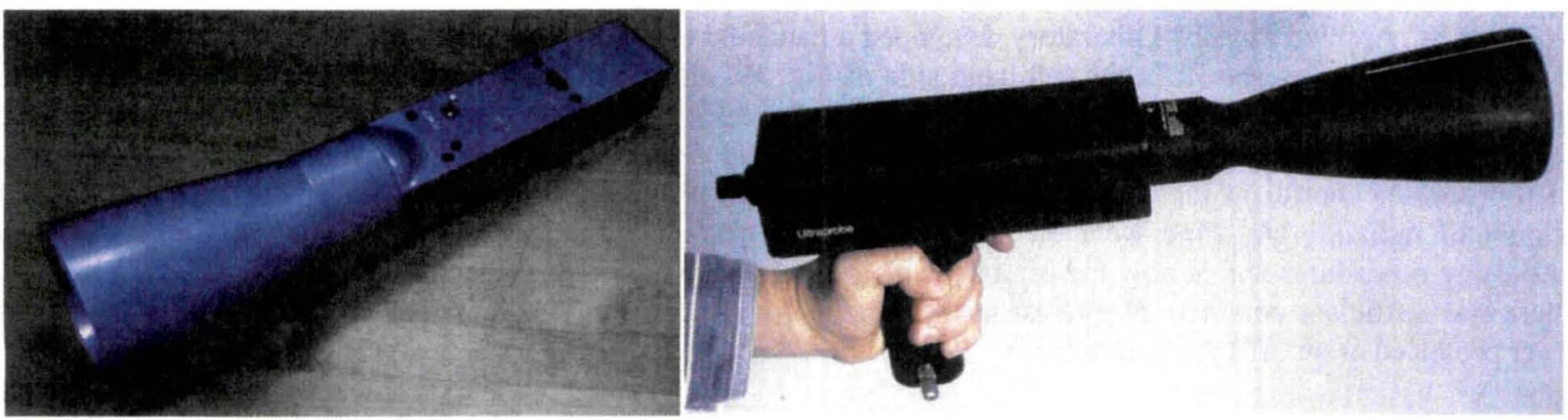

Figure 20. Ultrasonic Leak Detection with Parabolic Horns. Ultrasonic leak detectors were used to help locate hydrogen leaks in the Orbiter's aft compartment. Follow on work led to the development of a parabolic focusing horn that improved the collection efficiency and directionality of the state-of-the-art in this field. The left hand image shows the KSC developed instrument and the right hand image shows a commercial version of the horn attached to an ultrasonic leak detector.

The Orbiter Window Cavity Conditioning System (WCCS) supplies gaseous nitrogen during ascent to the cavities between the Orbiter window panes and this gas is kept clean and dry by a pair of parallel desiccant canisters, shown in Fig. 21. During re-entry to earth, air is drawn through the canisters to dry the air and prevent the Orbiter's windows from fogging up and potentially blocking the pilot's vision. There is a requirement that these canisters be checked after every flight to certify that they have not become plugged and that sufficient nitrogen or air can move through them. To perform this test a significant amount of equipment must be removed and handled, risking damage to not only the desiccant manifold but to the surrounding equipment. The Shuttle Ground Processing Team approached the KSC Applied Physics Laboratory and asked if there was a way that a flow measurement could be made without having to remove or disassemble any Orbiter hardware.

The KSC APL came up with a relatively simple solution to this problem that was quickly accepted by the Orbiter Processing Team ${ }^{26}$. A clip was designed that contained a heater and a temperature sensor as shown in Fig. 21. The Orbiter team was told to turn off the nitrogen flow and attach the clip to the line leading into or out of the desiccant canister. The heater would then warm up the line while the temperature was monitored and displayed on a computer screen. When the temperature stabilized the Orbiter team was instructed to turn on the nitrogen flow and observe the line temperature. The flowing nitrogen would cool down the line proportionately to the amount of flow. This concept was demonstrated to provide a reasonable indication of flow rate and thus the condition of the desiccant canister. So within a short time hardware was delivered and incorporated into the Orbiter processing flow. 


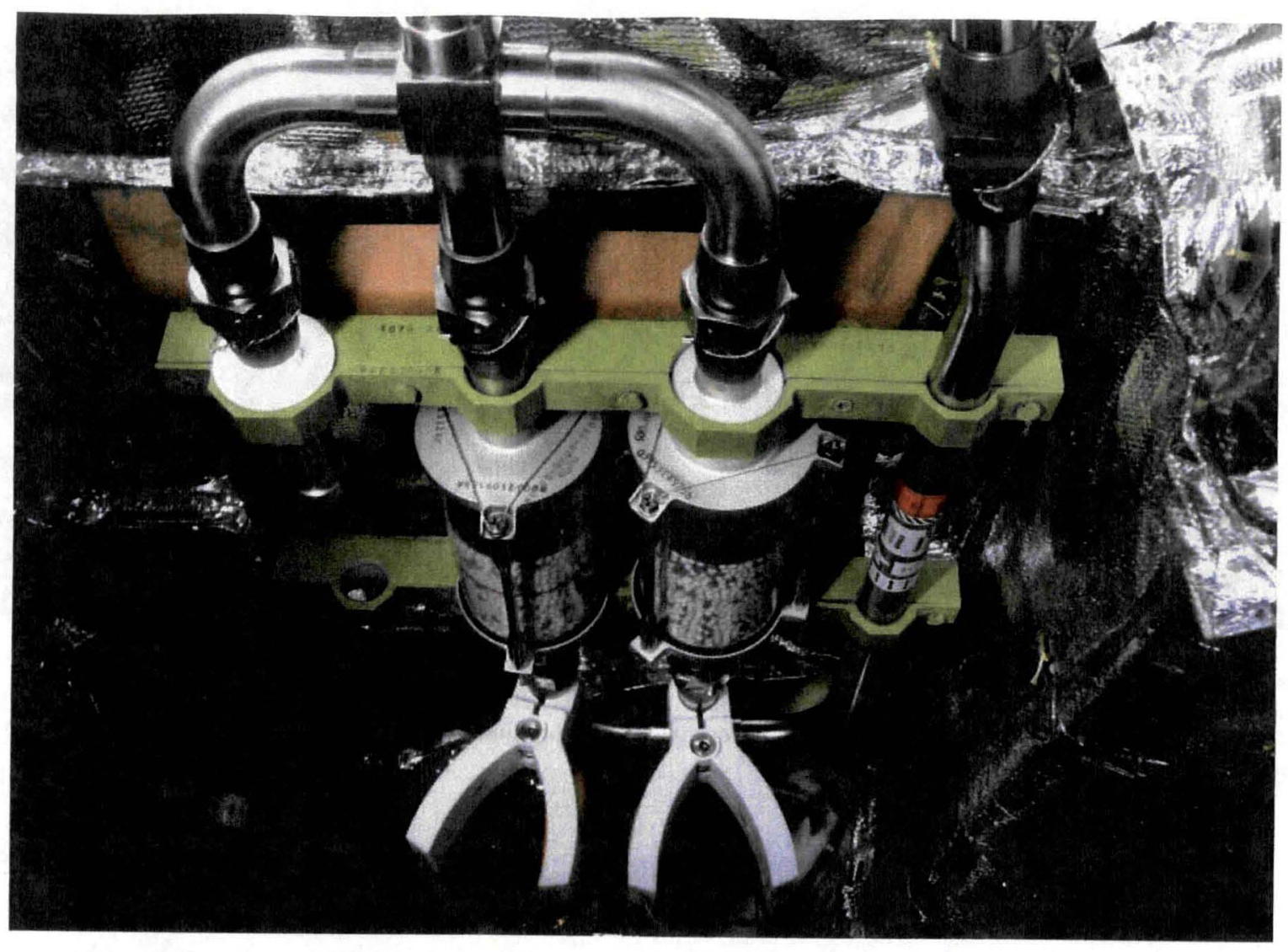

Figure 21. The WCCS desiccant canister flow detection system. This image shows the desiccant canisters used to clean and dry the air going to the Orbiter windows. The white clips are part of a system developed to ensure that there adequate gaseous nitrogen can move through the canisters.

The next problem brought to the KSC Applied Physics Laboratory was more serious. Within the payload bay of the Orbiter are a row of over-center latches that are used to hold the Orbiter's robotic arm in place during launch, but then allow it to be released when in orbit. These latches, one is shown in the left-hand image in Fig. 22, are two state mechanisms, where a center axis can be located on either side of an in-line position, in-line meaning lined up with axels at the top and bottom of the device. When the center axel is on one side of in-line it locks the latch down holding the robotic arm in place and when it is on the other side of in-line it releases the latch allowing the robotic arm to come free. This over-center latch has two primary adjustments; one controls the length of an arm of the latch, which then determines how much force is required to move the center axel through the in-line position, and one controls a stop determining how far past the in-line position the center axel can move. It is critical that these adjustments be made properly. If they are out of specification the robotic arm could come free during ascent resulting in a potential loss-of-mission or the motors that control the latch might not have enough torque to release the robotic arm resulting in a serious impact to a mission.

The problem with the over-center mechanism is that the drawing calls out for a specific distance that the center axel must be located past the in-line position, but there is no easy way to make this measurement. One might think that by drawing a line through the two outer axels that an in-line position can be found and a measurement made from this, but according to the drawing, nothing in this mechanism is machined to the precision required to make the measurement. The Shuttle team recognized that adjusting the over-center mechanism was not straightforward and after substantial effort came up with a controversial approach. They attached a precision position sensor and a strain gage to the mechanism to try and measure the position of the center axel when it was under maximal strain, assumed to be the in-line position. The problem is that the strain varies slowly with the distance past the in-line position, so this measurement has large errors. So the Shuttle Orbiter processing team brought this problem to the KSC Applied Physics Laboratory. 

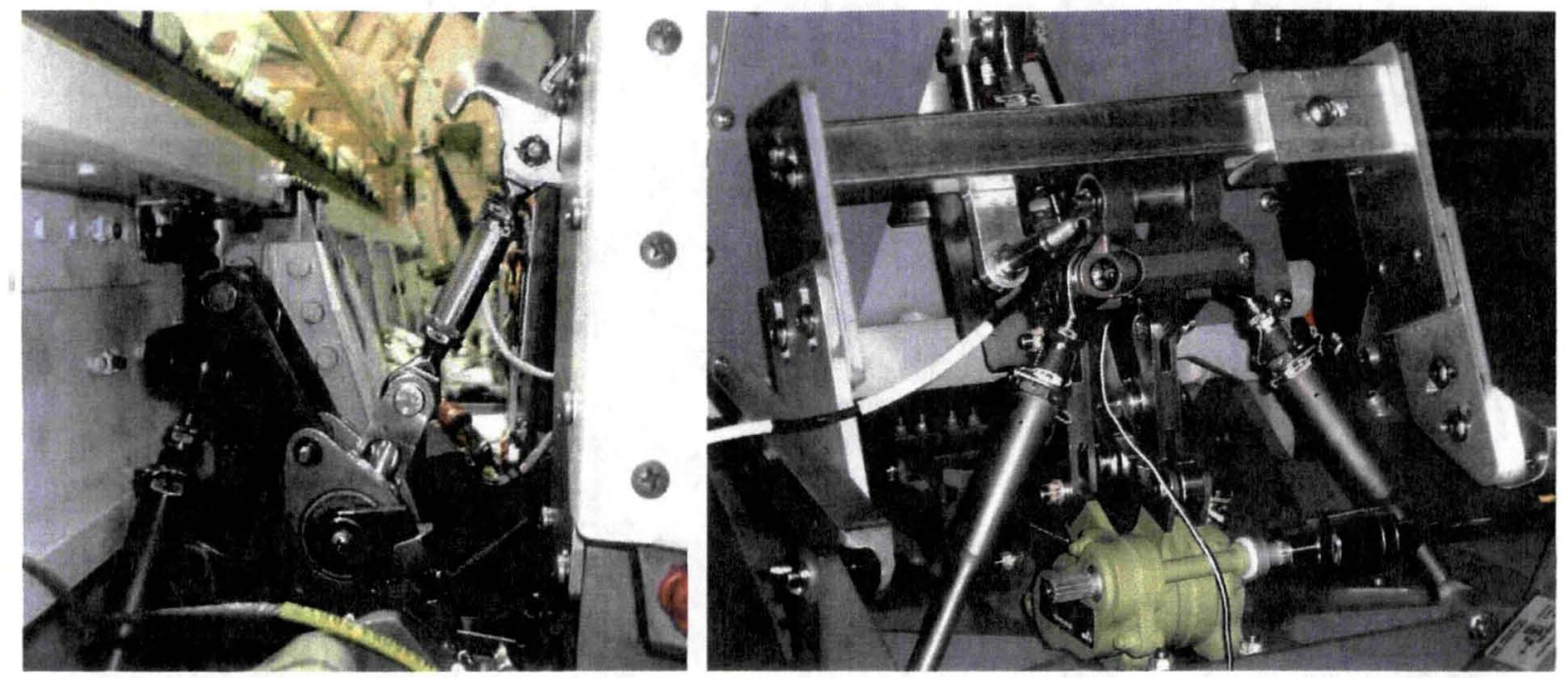

Figure 22. An approach for configuring an Orbiter payload bay over-center mechanism. The image on the left shows an over-center mechanism in the payload compartment of an Orbiter. This device is used to hold the Orbiter's robotic arm in place during launch. The image to the right shows a system designed to ensure that the over-center mechanism is adjusted to within correct launch specifications.

Solving the over-center mechanism measurement problem was not easy and some of our early attempts failed due to not understanding specific device details. However, we eventually solved it in a manner that not only supplied a measurement to the desired precision; it also required no new sensors, just a few additional brackets, including one, shown in the right-hand side of Fig. 22. This bracket holds a precision position sensor (the same one the Shuttle Program had previously obtained) against a plate attached to the over-center mechanism near the center axel. Now, turn the turn-buckle on the over-center mechanism so that the mechanism is lengthened and the center axel cannot be pushed through the in-line position. Next, apply a known force to the center axel (in our lab demo a calibrated torque wrench was used for this purpose), pushing it towards the in-line position and note the position reading. Then, pull the upper axel pin out, invert the over-center mechanism orientation into its other state, and slide the pin back in. Next, apply the same force to the center axle, but in the opposite direction, pushing it towards the inline position, but from the other side, and note the position reading. The average of the two position readings is the in-line position of the mechanism and all subsequent positions can be referenced to this value. This process is sufficiently accurate and precise to configure the over-center mechanism to launch specifications.

Another problem brought to the KSC Applied Physics Laboratory occurred during the return to flight effort after the Columbia accident, preparation for STS-114. While performing an External Tank filling test, Shuttle operations noticed that the External Tank ullage was not holding pressure properly. Normally, once the lower portion of the External Tank is filled with liquid hydrogen a vent is closed and the ullage is pressurized with pulses of gaseous helium. As the helium chills the pressure drops and additional pulses are added, but this is a well known process and if more than a specific number of pulses is required it is assumed that there is a problem somewhere. The first possibility is that the vent did not close properly and that it is leaking ullage gas into the vent line. So the Shuttle team requested a leak sensor that could be placed in this vent line, but it couldn't be more than an inch thick (that was all the room they could get by separating a nearby flange (see the right hand image in Fig. 23) and they needed it finished, tested, and ready to install in one week (before the next filling test).

A week later a Pitot tube based flow sensor was handed to the Shuttle team, see the left hand image in Fig. 23. A custom flange was cut from sheet steel and two small tubes were brought down into the center of the flow. One tube had an opening on its end, facing into the flow, so that the gas in it would increase in pressure with the flow rate and the other had an opening on its side so that the gas in it would decrease in pressure with increasing flow rate. The two tubes were accessible from outside of the flange and were monitored with a pair of pressure transducers, allowing a differential pressure measurement to be taken that was proportional to the flow rate. This hardware was 
designed, built, tested, and installed in an eight day period. It was used during the next filling test and, surprisingly, showed that the vent line was not leaking, that there was another reason for the ullage pressure decay.

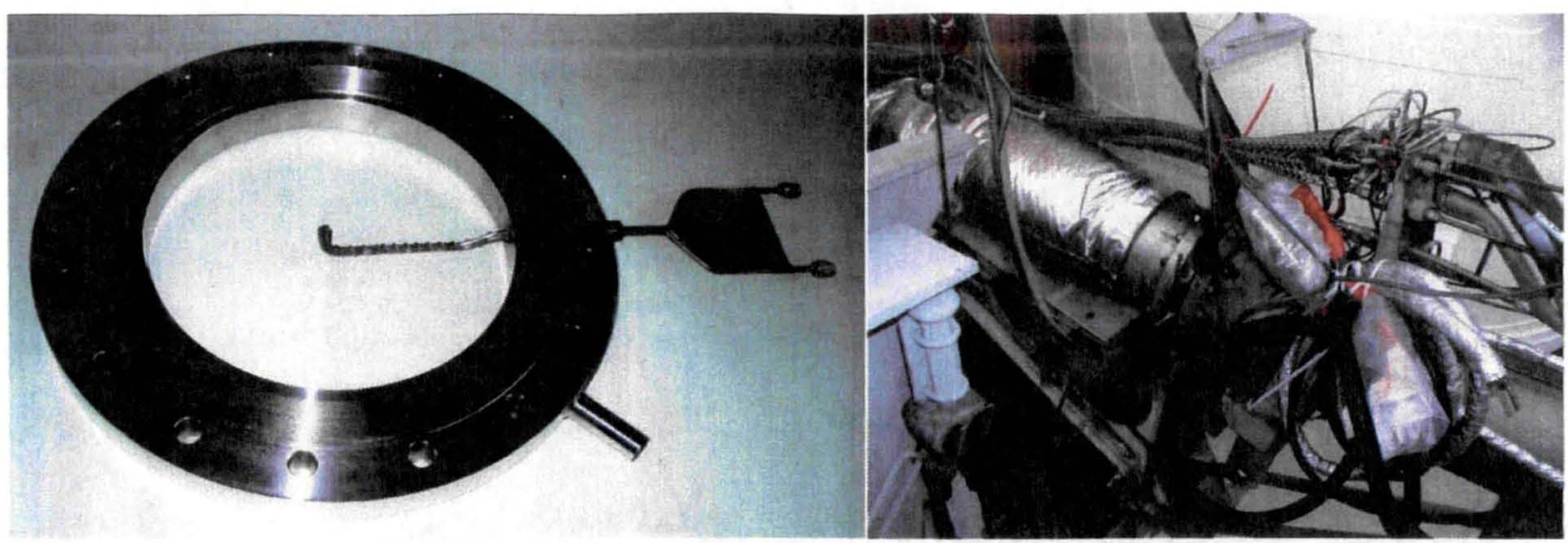

Figure 23. The External Tank vent-line leak monitoring system. The image on the left is a custom pitot-tube based sensor used to monitor the gas leakage from the External Tank. The image on the right is of the ET vent line with the sensor located in a gap between two of the flanges.

The launch of STS-114 proceeded and came very close to being scrubbed due to the ullage pressurization problem, so a 30 day study team was formed with support from the KSC Applied Physics Laboratory. The team found that there was significant uncertainty in the actual amount of helium used in pressurization. In other words, the problem might be that insufficient helium was being used for pressurization, not that there was a leak. After exploring several options the team decided to calibrate the small high pressure flow control orifice along with a section of upstream and downstream tubing as a make shift flow-meter. The KSC APL located a facility that could calibrate the inlet hardware, though due to costs dry air was used instead of helium. Analysis was then performed to determine the actual performance of the hardware yielding a more accurate determination of the flow per pulse which resolved the problem in time for STS-121.

Each launch pad has a pair of very large cryogenic tanks, one for liquid oxygen and one for liquid hydrogen (see the left hand image in Fig. 24). These tanks can hold as much as 800,000 gallons of liquid and were constructed in the 1960s in support of the Apollo Program. They are each composed of a pair of concentric steel spheres, the one on the outside is about 70 feet in diameter, made of carbon steel, and is exposed to the ambient environment-it is the sphere that is seen when looking at the tank and is usually coated or painted to protect it from corrosion. The inner sphere is about 4 feet smaller in radius, and is composed of stainless steel since it is in direct contact with the cryogenic commodity. For the hydrogen tanks the volume between these two spheres is under vacuum because air would liquefy and freeze against the inner tank if it were present. So even though there is no material present for heat conduction, heat can still move from the outer sphere to the inner one through radiation, so this volume is filled with a lightweight powder insulation called perlite. Analysis shows that an ideal tank, a tank with no issues, still has a small amount of heat leakage and should boil-off about 200 gallons of hydrogen per day. The Pad A tank has a boil-off rate of about this value and is representative of a well performing tank, but during the 1990s the Pad B tank boiled off as much as 800 gallons a day, far in excess of the design criteria.

The Shuttle Program approached the KSC Applied Physics Lab and asked for help in understanding this problem. It was soon determined that there was a cold spot on top of the tank (see the infrared image in Fig. 24) and observed that this explained why the area was kept wet enough that mold often grew there. Clearly there was a heat leak into the inner sphere in this area and the KSC Applied Physics lab, after some modeling, suggested that there was no perlite between the spheres in this location. Analysis backed up this theory and it soon became an accepted explanation of the problem, along with a belief that the tank had simply not been filled properly in the 1960s. There was some disagreement with this theory, with suggestions of water contamination in the perlite, but there was no analysis to back up this alternative. Later on, the Space Shuttle Program gave Launch Pad B to the Constellation Program. They drained the liquid hydrogen tank, slowly brought the structure up to ambient temperature, filled the inner tank region with dry nitrogen, and then opened the tank to look inside using remote cameras. They saw a large 
area under the cold spot where there was no perlite, in agreement with the KSC Applied Physics Lab prediction, and subsequently brought in additional perlite to fill this empty volume.

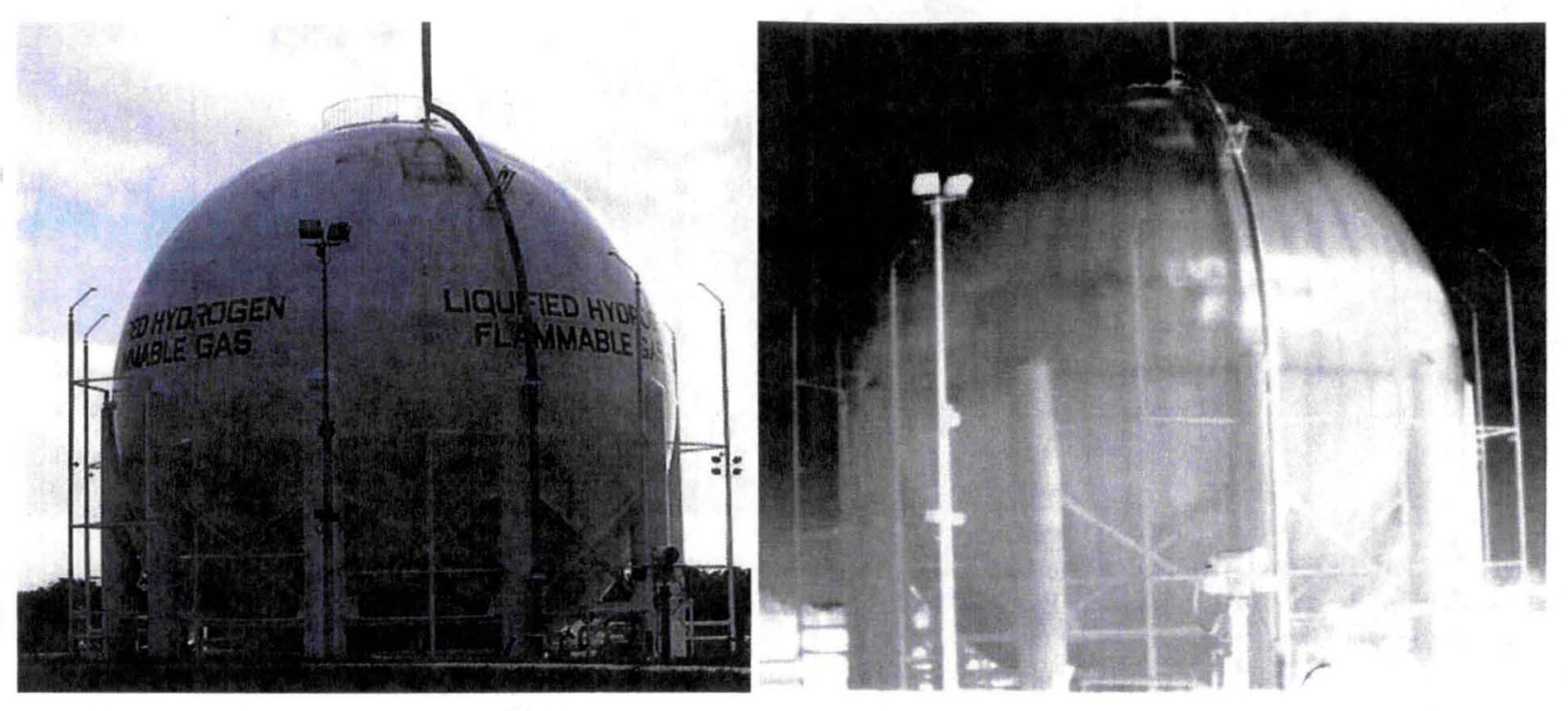

Figure 24. Visible and IR images of the Pad B liquid hydrogen storage tank. These are visible and infrared images of the Pad B liquid hydrogen storage tank. Note the large discoloration near the top indicative of a heat leak into the tank. This area is cold, as shown in the image on the right, and condenses water, causing it to remain wet and allowing mold to grow as seen in the image on the left.

Before leaving this topic, the KSC Applied Physics Laboratory put substantial effort into modeling and understanding these large cryogenic tanks. As a result of this we developed a technique using the sun as a heat source to allow the internal tank structures to be imaged with an infrared camera. In the infrared image in Fig. 24 the internal ribbing can be seen as dark, i.e., cool areas. The lab developed a sensor to help determine if the perlite within the tank was compacted on bottom of the tank ${ }^{27}$. This sensor showed no sign of compaction and a subsequent perlite test (performed after the tank was warmed up and the vacuum removed) agreed with this assessment. Finally, the lab showed that the first sign of an air leak into the inner tank perlite volume would be an increase in boil-off rate caused by the freezing of this air against the inner tank causing heat transfer to it. Analysis was performed to equate boil-off with the volume of an air leak and an improved set of pressure transducers was obtained with the intent of improving the monitoring of boil-off and thus of issues such as air leaks. Overall the KSC Applied Physics Lab greatly improved the understanding of these tanks, helping the Shuttle Program deal with and address various issues that had arisen over their years of service.

\section{E. Reaching Beyond the Lab}

There have been occasions where problems have been brought to the KSC Applied Physics Laboratory that required expertise unavailable within our team. In some of these cases the problems were turned over to other labs within the Kennedy Space Center. This occurred with problems within the areas of cryogenics, OEM sensors, purely mechanical design, electrostatics, corrosion, chemical, polymer, RF, and other areas where developed expertise exists within the center. In many cases the lab generated technology prototypes and then other groups would design and implement the field version of the hardware. One example of this was the gaseous oxygen (GOX) vent hood camera system designed to monitor the top of the External Tank, looking through the windows on the GOX vent hood, for the presence of ice build-up. Another was the Orbital Maneuvering System (OMS) pod alignment tool, a tool designed to align two holes to a thousandth of an inch tolerance so that a bolt could be pushed through both holes to interface with a floating nut. In both cases the KSC APL designed and constructed the first version of the hardware and another group converted it to a final version. There was also a case where an electrical connector alignment tool was requested and the KSC APL built a laser version and asked a design group to build a mechanical 
version. Then, a head-to-head evaluation was carried out and the mechanical version was chosen to be converted to a field tool. Yet, in all of these cases, the KSC APL was the contact point for the Shuttle Program and generated the concepts and prototypes needed to eventually achieve solutions to these problems.

The main emphasis of this section is on two cases where state-of-the-art technology, beyond that available at the Kennedy Space Center, was needed in order to meet a Shuttle requirement. In both cases the lab determined that the best way to proceed would be through the Small Business Technology Transfer Research Program (STTR) where a small business is funded to help move a university developed technology from a laboratory to a commercial product. Also, in both cases, Graduate Student Research Program (GSRP) funding was obtained to help supplement the university portion of the effort with graduate student support. The two cases to be discussed below represent a true legacy of the Shuttle Program in that novel technology was developed to meet a Shuttle need, but where that advance also has potential to affect commercial industry.

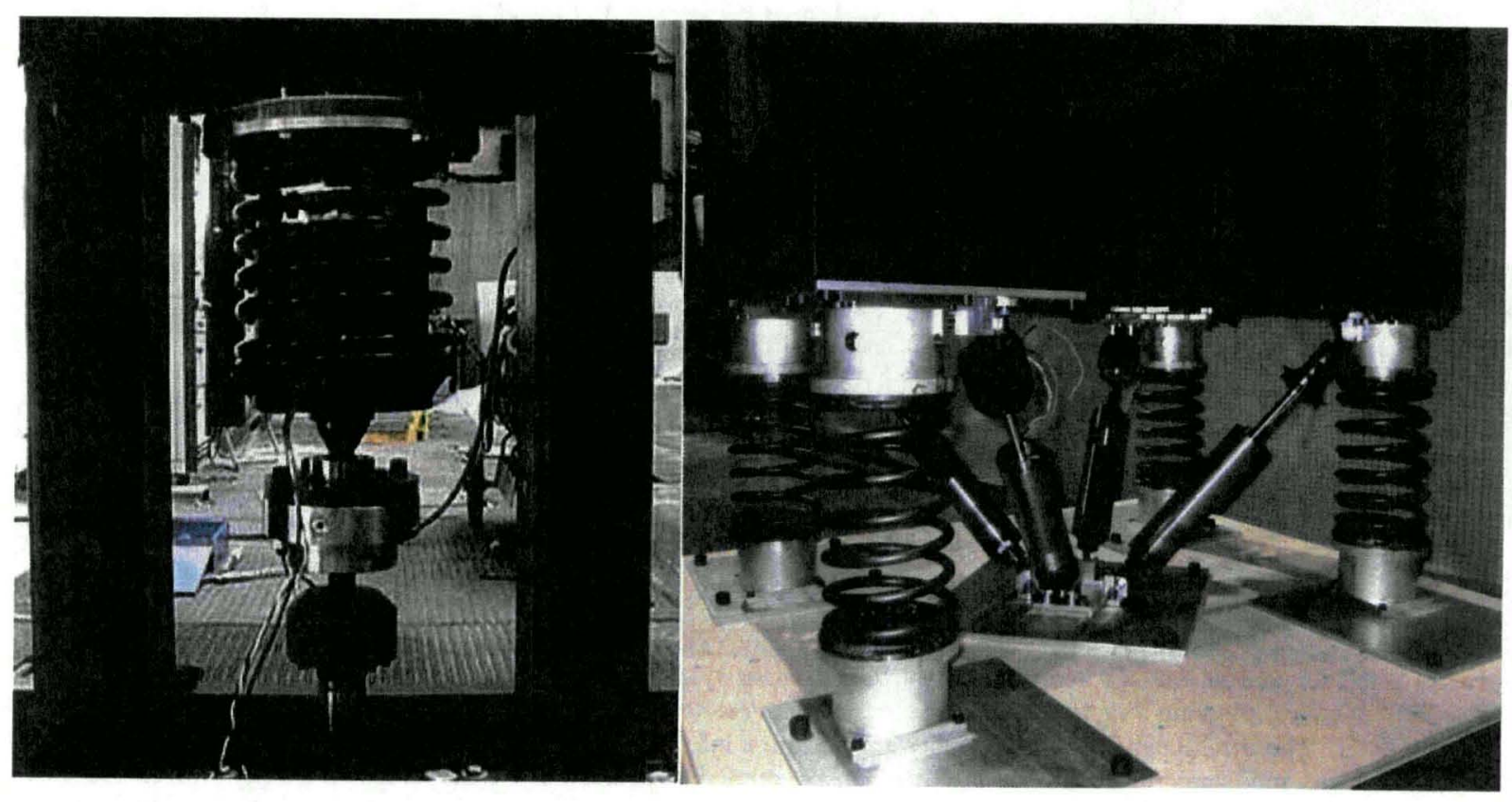

Figure 25. Active Vibration Isolation of racks in the mobile launch platform. Two different approaches to isolating sensitive rack mounted equipment from launch shock/vibration are shown. To the left is a Balcones Technologies, LLC,-University of Texas at Austin, direct-drive, voice-coil, electromagnetic actuator supplying direct force compensation to minimize the vibration seen by the rack. This approach provides a high degree of vibration isolation, but is heavier and more expensive. To the right is a Techno-Science Inc.-University of Maryland adaptive magnetorheological isolator that operates by electrically controlling damping. This approach does not supply the same degree of vibration isolation, but is lighter and less expensive.

When the Space Shuttle launches, the impact of the rocket plumes causes significant shock and vibration, often resulting in damage to sensitive rack mounted equipment located within the mobile launch platform (MLP). In an effort to reduce this damage a spring mounted floor is used to isolate some of this equipment, but this approach has not always protected the hardware. A few years ago the KSC Applied Physics Laboratory suggested that it might be worth considering upgrading this passive isolation system with an active one and the Shuttle Program agreed. As a consequence an STTR call went out and two different active vibration isolation approaches were proposed and Phase I and Phase II contracts were awarded, funding both of these. In the first approach Balcones Technologies, LLC, and the University of Texas at Austin, Center for Electromechanics developed a direct force compensation electromagnetic actuator ${ }^{28}$ that drives the rack in the opposite direction of the floor motion, canceling out the MLP motion. This approach provides significant vibration isolation, but the actuator is heavy and expensive. In the second approach Techno-Science Inc. and the University of Maryland developed an adaptive magnetorheological isolator ${ }^{29}$ that operates by electrically controlled damping. This approach, commonly used on luxury automobiles, can substantially block vibration and shock, but not to the same degree as the direct force compensation approach, yet it 
is lighter and less expensive. Both approaches have been recently demonstrated (see Fig. 25) and represent an advance in this technology area. Sadly, the Shuttle Program ended before it could take advantage of this technology advance, but interest has been shown by potential follow-on programs. Even so, this Shuttle directed work has advanced the state-of-the-art in the field of active vibration isolation and will impact this field into the future.
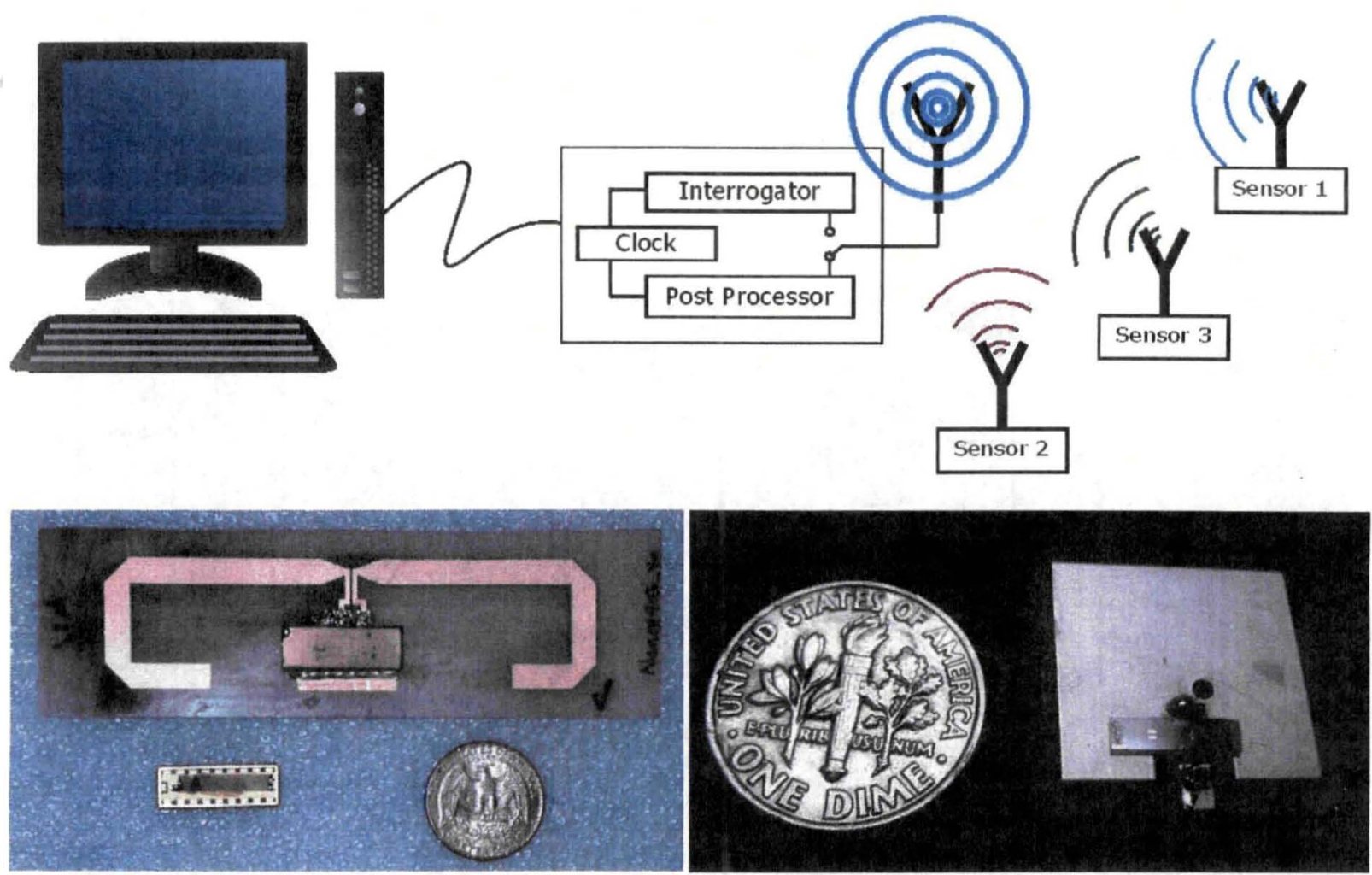

Figure 26. Wireless passive surface acoustic wave (SAW) sensor systems. The upper image shows a sketch of how a wireless sensor system operates, showing a radio frequency pulse being transmitted to a set of sensors that generate echo patterns detected by the transmitter. These echo patterns supply sensor identification information as well as the measurement information. The left lower image shows a moderate range version of a SAW sensor coupled to an antenna and the image on the right lower side shows a short range version.

Several years ago the Shuttle Program requested a passive, wireless sensor that could be installed in the Orbiter's tiles to provide information on their health. The hope was that internal damage and adhesion problems could be detected early, but the request was extreme relative to the state-of-the-art on several fronts. The sensors would need to be very small to not impact the tile performance; they would need to be wirelessly interrogated individually in order to know which tile was being monitored; they would need to be passive since batteries could not be placed into the tiles, and they would need to be able to survive the reentry heat conditions. Even so, an STTR call went out and several company/university teams responded and were funded. After the Phase I efforts were complete it was clear that the best chance of success was to move forward with wireless surface acoustic wave (SAW) sensors. These devices are radio frequency (RF) transducers where an incoming RF pulse excites a surface acoustic wave that reflects off of periodic structures, resulting in a series of reflected RF pulses that both identify the sensor and supply a measurement ${ }^{30,31}$.

After substantial work, multiple sensors were created including temperature, pressure, liquid level, hydrogen, humidity, etc. and then, during the summer of 2010, a multiplexed four sensor system was demonstrated by the University of Central Florida working with Mnemonics, Inc. In this system, see Fig. 26, a transmitter/receiver launches an RF pulses and the sensors produce their unique reflection patterns that are detected and then processed with the results displayed on a computer. The lower images in Figure 26 show a long range device on the left hand side and a short range device on the right hand side. The long range device has been shown to communicate with the 
transmitter/receiver out to 60 meters, while the short range device has been developed for applications where small size is important. Already, a major aerospace company has decided to invest in this technology as a way to monitor a number of parameters on previously inaccessible objects, such as rotors or engine components.

There is significant excitement about wireless SAW sensing systems. The sensors are inexpensive, can survive excessive temperature ranges, are radiation hardened, and should operate for many years. They can be located in concrete, walls, or spacecraft. They can operate in oil wells or on the surface of the moon. But they may also become ubiquitous, being installed in our homes, our cars, and numerous structures, allowing our cell phones or computers to communicate with them and continuously monitor the world within which we live. This technology, a direct result of a request from the Shuttle Program, may have more long term impact on mankind than any other legacy item from the program.

\section{Conclusion}

The primary conclusion of this paper is that a technology infusion group, or innovation support lab, needs to be located near any major program that has a long duration. As time passes requirements will change, new methods and technologies will become available, and new problems will emerge. An independent group is needed to help solve these problems, presenting solutions to the program. This lab should not have any actual authority, decisions should be made by the program, and the lab should to some extent sink or swim on its own achievements. They should be a service provider to the program, treating the program as their customer, listening rather than dictating. They should represent as broad a background as possible in order to minimize the natural tendency to force an area of expertise onto a problem. Ideally they should have access to a wide range of experts in related fields so they can disperse problems, but they should still keep track of the individual customers. Also, they must be in close proximity to the program so that there can be face-to-face meetings, the actual hardware can be seen, the technicians can be involved, and permitting organizations such as safety and quality can be involved in the development.

The impact that such a lab, namely the KSC Applied Physics Laboratory, has had on the KSC Shuttle Ground Processing Directorate, has been partially described in the examples given above, but this is only part of the story. For every piece of delivered hardware there are roughly two that did not make it into the field for some reason, yet the technology development and innovation was still there and affected the program. Simply showing the program engineers and technicians that innovation was possible often led them to approach the lab with problems, sometimes years later, or to move forward with their own solutions. The true legacy of the lab is that it fostered an environment of innovation, setting an example, providing encouragement, distributing work, and helping to continually make ground processing of the Space Shuttle safer, more efficient, and in some cases, possible.

\section{Acknowledgments}

First and foremost, the past and present members of the KSC Applied Physics Laboratory need to be acknowledged; Ellen Arens, Bradley Burns, Janine Captain, Robert Cox, Mike Csonka, Adam Dokos, Greg Hall, William Haskell, Curtis Ihlefeld, John Lane, Teresa Lawhorn, Frank Lorenzo-Luaces, Mark Minich, Steve Moerk, Tom Moss, Kevin Murtland, Mark Nurge, Steve Parks, Jim Polk, Ken Rose, Stanley Starr, Steve Simmons, Jim Strobel, Douglas Willard, and Robert Youngquist. Then, working alongside of this group were numerous NASA and contractor specialists who contributed to the lab products. In addition, of course, the Space Shuttle Ground Processing engineers, managers, and technicians need to be acknowledged for bringing problems to the lab's attention, for working with the lab on solutions, and then for accepting these solutions and helping to insert them into the ground processing system.

Special recognition is acknowledged to Charles Stevenson. His vision to utilize the research and development expertise of the KSC Applied Physics Laboratory to solve Space Shuttle Program's problems provided the driving force for many of these innovations. 


\section{References}

${ }^{1}$ Collins, J. D., et al., "Optoelectronic Alignment and Ultrasonic Ranging Apparatus," Federal Lab Laser Tech Briefs, Vol. 3 , No. 1, February 1995 , pp. 16.

${ }^{2}$ Burris, D. E.., et al., "Using Laser rangefinders to Align Two Structures," NASA Tech Briefs, Photonics Tech Briefs Special Supplement, Vol. 25, No. 1, January 2001, pp. 16a.

${ }^{3}$ Youngquist, R.C., et al. "Optoelectronic System for Measuring Heights Above a Floor," NAS Tech Briefs, Photonics Tech Briefs Special Supplement, Vol. 27, No. 3, March 2003, pp. 8a-10a.

${ }^{4}$ Strobel, J. P., Polk, J. D., Haskell, W. D., and Youngquist, R. C., “Apparatus and Technique for Measuring Distance Between Axles," NASA Tech Briefs, Vol. 24, No. 3, March 2000, pp. 76.

${ }^{5}$ Stevenson, C. G., Rivera, J. E., Youngquist, R. C., Cox, R. B., and Haskell, W. D., "Optoelectronic Tool Adds Scale marks to Photographic Images," NASA Tech Briefs, Photonics tech Briefs Special Supplement, Vol. 27, No. 1, January 2003, pp. 6a-8a.

${ }^{6}$ Rivera, J. E., Youngquist, R. C., Cox, R. B., Haskell, W. D. and Stevenson, C. G., United States Patent, "Scaling Device for Photographic Images,” Number 6,891,148, May 10, 2005.

${ }^{7}$ Braden, B. M., Youngquist, R. C., Cox, R. B., and Floyd, C. R., "Illumination Device for Inspecting Window," NASA Tech Briefs, Vol. 22, No. 11, November 1998, pp. 68-70.

${ }^{8}$ Tiziani, H. J. and Uhde, D. M., "Three-Dimensional Image Sensing by Chromatic Confocal Microscopy," Applied Optics, April 1994, Vol. 33, No. 10, pp. 1838-1843.

${ }^{9}$ Ihlefeld, C. M., Dokos, A. G., and Burns, B. M., "Portable Handheld Optical Window Inspection Device," NASA Tech Briefs, Vol. 34, No. 11, November 2010, pp. 36.

${ }^{10}$ Columbia Accident Investigation Board (CAIB) Report, Appendix E.2 contains NSTS-37384, STS-107 Image Analysis Team Final Report in support of the Columbia Accident Investigation, June 302003.

${ }^{11}$ Klinko, S., Lane, J. E. and Nelson, C. , "Measuring Positions of Objects Using Two or More Cameras," NASA Tech Briefs, Vol. 32, No. 6, June 2008.

${ }^{12}$ Metzger, P. T., Lane, J. E., Carilli, R. A., Long, J. M., and Shawn, K. L., "Photogrammetry and Ballistic Analysis of a High-Flying Projectile in the STS-124 Space Shuttle Launch," Acta Astronautica, Vol. 67, July-August 2010, pp. $217-229$.

${ }^{13}$ Youngquist, R. C., "Water Detection and Removal From Shuttle Tiles," AIP Space Technology and Applications Int. Forum-STAIF 2003 Conference, Vol. 654, January 28, 2003, pp. 269-275.

${ }^{14}$ Youngquist, R. C., Surma, J., and Parks, S. L. , "Hygrometer for Detecting Water in Partially Enclosed Volumes," NASA Tech Briefs, Vol. 29, No. 12, December 2005.

${ }^{15}$ Gegoris, D. J., United States Patent, "Electro-Optic Ice Detection," Number 5,500,530. March 19, 1996.

${ }^{16}$ Youngquist, R. C., Haskell, W. D., Immer, C., Cox, R. B., and Lane, J. E. , "Hail Monitor Sensor," NASA Tech Briefs, Vol. 33, No. 7, July 2009.

${ }^{17}$ Lane, J. E., Youngquist, R. C., Haskell, W. D., Immer, C., and Lane, J. E. , "A Hail Size Distribution Impact Transducer," Journal of the Acoustical Society of America Express Letters, Vol. 119, Issue 3, February 2006, pp. EL47-EL53.

${ }^{18}$ Hall, G.A., et al., "Ultraviolet Source for Testing Hydrogen-Fire Detectors," NASA Tech Briefs, Laser Tech Briefs Supplement, Vol. 19, No. 8, August 1995, pp.12a-13a.

${ }^{19}$ Youngquist, R. C., Moerk, J. S., and Strobel, J. P., United States Patent, "Portable Light Source for Simulating Fires having an Adjustable Aperture," Number 5,627,362, May 6, 1997.

${ }^{20}$ Hall, G.A., et al., "Hydrogen-Fire-Detector Calibration Unit," NASA Tech Briefs,, Vol. 21, No. 10, October 1997, pp.46-48.

${ }^{21}$ Strobel, J. P., Moerk, J. S., and Youngquist, R. C.,, United States Patent, "Optical Detector Calibrator System," Number $5,561,290$, October 1, 1996.

${ }^{22}$ Youngquist, R.C., et al., "Improved Portable Ultrasonic Leak Detectors," NASA Tech Briefs, Vol. 19, No. 2, February 1995, pp. 27.

${ }^{23}$ Youngquist, R. C., and Moerk, J. S., United States Patent, "Ultrasonic Leak Detection System," Number 5,710,377, January 20, 1998.

${ }^{24}$ Haskell, W. D., Strobel, J. P., Youngquist, R. C., Moerk, J. S., and Cox, R. B., "Imaging with a Focal-Plane Array of Ultrasonic Transducers," NASA Tech Briefs, Vol. 24, No. 4, April 2000, pp.35-36.

${ }^{25}$ Youngquist, R. C., and Moerk, J. S., United States Patent, "Ultrasonic Imaging System," Number 5,979,239, Nov. 9, 1998.

${ }^{26}$ Moss, T. J., Ihlefeld, C. M., and Slack, B. C., "Gas Flow Detection System," NASA Tech Briefs, Vol. 24, No. 4, April 2000, pp.35-36.

${ }^{27}$ Youngquist, R. C. , "Method for Detecting Perlite Compaction in Large Cryogenic Tanks," NASA Tech Briefs, Vol. 34 , No. 4, April 2010, pp.21-22.

${ }^{28}$ Weeks, D. A., Bresie, D. A., Beno, J. H. and Guenin, A. M., "The Design of an Electromagnetic Linear Actuator for an Active Suspension," SAE International Congress \& Exposition, Session: Steering \& Suspension Technology Symp., March 1999.

${ }^{29}$ Hiemenz, G. J., Hu, W., and Wereley, N. M., "Adaptive Magnetorheological Seat Suspension for the Expeditionary Fighting Vehicle," Journal of Physics: Conference Series, Vol. 149, No. 1, 2009.

${ }^{30}$ Malocha, D. C., Puccio, D., and Gallagher, D. , "Orthogonal Frequency Coding for SAW Device Applications," Ultrasonics Symposium, 2004 IEEE, Vol. 2, August 2004. pp. 1082-1085.

${ }^{31}$ Kozlovski, N. Y. and Malocha, D. C., "SAW Passive Wireless multi-Sensor System," Ultrasonics Symposium, 2009 IEEE International, September 2009. pp. 1541-1544. 